\title{
ON THE BOSONIZATION OF THE SUPER JORDAN PLANE
}

\author{
NICOLÁS ANDRUSKIEWITSCH, DIRCEU BAGIO, SARADIA DELLA FLORA, \\ DAIANA FLÔRES
}

Dedicated to Professor Ivan Shestakov on the occasion of his 70th birthday

\begin{abstract}
Let $H$ and $K$ be the bosonizations of the Jordan and super Jordan plane by the group algebra of a cyclic group; the algebra $K$ projects onto an algebra $L$ that can be thought of as the quantum Borel of $\mathfrak{s l}(2)$ at -1 . The finite-dimensional simple modules over $H$ and $K$, are classified; they all have dimension 1 , respectively $\leq 2$. The indecomposable $L$-modules of dimension $\leq 5$ are also listed. An interesting monoidal subcategory of $\operatorname{rep} L$ is described.
\end{abstract}

\section{Contents}

1. Introduction

2. The quantum Borel subalgebra of $\mathfrak{s l}(2)$ at -1

3. The bosonization of the Jordan plane 15

4. The bosonization of the super Jordan plane 17

References 23

\section{INTRODUCTION}

In this paper we start the study of the representation theory of two Hopf algebras introduced in $\mathrm{AAH} 1$ and $\mathrm{AAH2}$. In this last paper, Nichols algebras over abelian groups with finite Gelfand-Kirillov dimension were classified under some suitable hypothesis. The list includes the well-known Jordan plane denoted here $\mathcal{A}$ and a new Nichols algebra called the super Jordan plane denoted here $\mathcal{B}$. The study of various aspects of $\mathcal{B}$ was undertaken in $\mathrm{ABDF}, \mathrm{RS}$. Let $G$ be an infinite cyclic group denoted multiplicatively with a fixed generator $g$. The Nichols algebras $\mathcal{A}$ and $\mathcal{B}$ are realized in ${ }_{\mathbb{k} G}^{\mathbb{k} G} \mathcal{Y} \mathcal{D}$ and we have then the Hopf algebras $H=\mathcal{A} \# \mathbb{k} G$ and $K=\mathcal{B} \# \mathbb{k} G$, where \# stands for the Radford-Majid bosonization. The liftings or deformations of $H$ and $K$ were computed in AAH1.

2000 Mathematics Subject Classification. 16G30,16G60, 16 T05.

N. A. was partially supported by CONICET, MATHAMSUD, Secyt (UNC).

D. B. and D. F. were supported by FAPERGS 2193-25.51/13-3, MATHAMSUD. 
The purpose of this paper is to begin the study of the tensor category of $K$-modules. For this it is useful to study the $H$-modules, since $H$ embeds into $K$, and also the $L$-modules, where $L$ is the quantum Borel of $\mathfrak{s l}(2)$ at -1 that appears as a quotient of $K$. We obtain:

- The classification of the simple objects in rep $K$, that reduces to those in rep $L$, see Proposition 2.1 and Theorem 4.3. all have dimension 1 or 2 .

- The classification of the simple objects in rep $H$, all of dimension 1 , and of those indecomposable of dimension 2, see Propositions 3.3 and 3.4

- The classification of the indecomposable objects in $\operatorname{rep} L$ of dimensions $\leq 5$, see Propositions 2.15, 2.16, 2.17 and 2.18.

- An interesting subcategory of rep $L$, see Subsection 2.4.

1.1. Notations and conventions. We denote the natural numbers by $\mathbb{N}$ and $\mathbb{N}_{0}=\mathbb{N} \cup 0$. If $k<t \in \mathbb{N}_{0}$, then we denote $\mathbb{I}_{k, t}=\left\{n \in \mathbb{N}_{0}: k \leq n \leq t\right\}$, and $\mathbb{I}_{t}:=\{1, \ldots, t\}$.

We work over an algebraically closed field $\mathbb{k}$ of characteristic 0 . The group of $n$-th roots of $1 \mathrm{in} \mathbb{k}$ is denoted $\mathbb{G}_{n} ; \mathbb{G}_{n}^{\prime}$ is the subset of the primitive ones.

We denote by $e_{1}, \ldots, e_{n}$ the canonical basis of $\mathbb{k}^{n}$, which is identified with the space of column vectors. Given a vector space $V, T \in$ End $V$ and $\lambda \in \mathbb{k}$, we denote $V_{T}^{\lambda}=\operatorname{ker}(T-\lambda)$ and $V_{T}^{(\lambda)}=\cup_{j \in \mathbb{N}} \operatorname{ker}(T-\lambda)^{j}$. The Jordan block of size $r$ associates to $\lambda$ is denoted by $\mathrm{J}_{r}(\lambda)$. The set of eigenvalues of $T$ is denoted $\operatorname{spec} T$.

We write $X \leq Y$ to express that $X$ is a sub-object of $Y$ in a category $\mathfrak{C}$. All modules are left modules. As usual, $\operatorname{rep} A$ is the category of finitedimensional representations of an algebra $A$; we use indistinctly the languages of representation and module theories. The braided tensor category of left Yetter-Drinfeld modules over a Hopf algebra $H$ is denoted by ${ }_{H}^{H} \mathcal{Y} \mathcal{D}$.

\section{The Quantum Borel Subalgebra of $\mathfrak{s l}(2)$ AT -1}

Let $\mathcal{R}=\mathbb{k}[y]$ be the polynomial algebra in one variable. We realize $\mathcal{R}$ as a Hopf algebra in ${ }_{\mathbb{k} G}^{\mathbb{k} G} \mathcal{Y} \mathcal{D}$ by declaring $\mathcal{R}_{g^{n}}=\mathbb{k} y^{n}, g \cdot y^{n}=(-1)^{n} y^{n}$ and $y$ to be primitive. Let $L=\mathcal{R} \# \mathbb{k} G$ the bosonization of $\mathcal{R}$ by $\mathbb{k} G$; i. e. $L$ is the algebra generated by $y$ and $g^{ \pm 1}$ with relations $g^{ \pm 1} g^{\mp 1}=1$,

$$
g y g^{-1}=-y .
$$

The comultiplication, the counit and the antipode of $g$ and $y$ are determined by

$$
\Delta(g)=g \otimes g, \quad \Delta(y)=y \otimes 1+g \otimes y .
$$

The Hopf algebra $L$ is the quantum Borel subalgebra of $\mathfrak{s l}(2)$ at -1 . Let $L^{(n)}=L /\left\langle g^{2 n}-1\right\rangle$, a quotient Hopf algebra of $L, n \in \mathbb{N}$. The subalgebra $\mathbb{k}\left\langle g^{2}, y^{2}\right\rangle$ is a central Hopf subalgebra, the ideal $I$ generated by $g^{2}-1$ and $y^{2}$ is a Hopf ideal and $L / I$ is the 4 -dimensional Sweedler algebra $H_{4}$. Thus 
we have exact sequences of Hopf algebras

$$
\mathbb{k}\left\langle g^{2}, y^{2}\right\rangle \hookrightarrow L \rightarrow H_{4}, \quad \mathbb{k}\left\langle g^{2 n}\right\rangle \hookrightarrow L \rightarrow L^{(n)} .
$$

The Hopf algebras $L$ and $L^{(n)}$ are pivotal and $L^{(1)}$ is spherical.

2.1. Simple $L$-modules. We state here the (probably well-known) classification of the finite-dimensional simple $L$-modules; we give a proof for completeness. Throughout this subsection, $V \in \operatorname{rep} L$.

Let $a \in \mathbb{k}^{\times}$be an eigenvalue of $g$ and $V^{a}=V_{g}^{a}$. Then $y V^{a} \subset V^{-a}$, thus $V^{a} \oplus V^{-a}$ is a nonzero $L$-submodule of $V$. Also, $y V_{g}^{( \pm a)} \subset V_{g}^{(\mp a)}$.

We describe the one-dimensional representations of $L$. Given $a \in \mathbb{k}^{\times}$, let $\mathbb{k}_{a}=\mathbb{k}$ with the action $y \cdot 1=0, g \cdot 1=a$. Clearly $\mathbb{k}_{a} \in \operatorname{rep} L, \mathbb{k}_{a} \simeq \mathbb{k}_{b}$ if and only if $a=b$, and every one-dimensional representation of $L$ is like this.

Next we describe the two-dimensional irreducible representations of $L$. Given $a, b \in \mathbb{k}^{\times}$, let $\mathcal{U}_{a, b}=\mathbb{k}^{2}$ with the representation

$$
g \mapsto\left(\begin{array}{cc}
a & 0 \\
0 & -a
\end{array}\right), \quad \quad y \mapsto\left(\begin{array}{cc}
0 & b \\
1 & 0
\end{array}\right) .
$$

It is easy to check that $\mathcal{U}_{a, b} \in \operatorname{rep} L$ is irreducible.

Proposition 2.1. Let $V$ be a finite-dimensional simple $L$-module. Then either $V \simeq \mathbb{k}_{a}$ for a unique $a \in \mathbb{k}^{\times}$, or else $V \simeq \mathcal{U}_{a, b}$ for some $a, b \in \mathbb{k}^{\times}$. Moreover, $\mathcal{U}_{a, b} \simeq \mathcal{U}_{a, c}$ if and only if $b=c$.

Proof. First we prove that $\operatorname{dim} V \leq 2$. Let $a$ be an eigenvalue of $g$. Since $V^{a}$ is invariant by $g$ and $y^{2}$ and $g y^{2}=y^{2} g$, there exists $v \in V^{a}$ which is a common eigenvector of $g$ and $y^{2}$. Then the subspace $\langle v, y v\rangle$ of $V$ is a non-trivial $L$-submodule of $V$. Thus $V=\langle v, y v\rangle$.

Assume that $\operatorname{dim} V=2$. We claim that $y$ is invertible. Indeed, ker $y$ is an $L$-submodule of $V$. If $\operatorname{ker} y=V$ and $v$ is an eigenvector of $g$, then $\langle v\rangle \leq V$, a contradiction. Thus ker $y=0$. Now $g$ has exactly two eigenvalues $a$ and $-a$. If $v \in V^{a}, v \neq 0$, then $y v \in V^{-a}$ and $\{v, y v\}$ is a basis of $V$. Suppose that $y^{2} v=b v+b^{\prime} y v$, where $b, b^{\prime} \in \mathbb{k}$. By (2.1) $b^{\prime}=0$. Hence $V \simeq \mathcal{U}_{a, b}$. It is straightforward to check that $\mathcal{U}_{a, b} \simeq \mathcal{U}_{a, c}$ if and only if $b=c$.

Remark 2.2. As is well-known, Proposition 2.1 extends to $q \in \mathbb{G}_{n}^{\prime}$ instead of -1. Indeed, let $L_{q}=\mathbb{k}\left\langle y, g^{ \pm 1} \mid g^{ \pm 1} g^{\mp 1}-1, g y g^{-1}-q y\right\rangle$, the quantum Borel of $\mathfrak{s l}(2)$ at $q$. Then any simple object of $\operatorname{rep} L_{q}$ is isomorphic either to $\mathbb{k}_{a}$, or else to $\mathcal{U}_{a, b}$ for some $a, b \in \mathbb{k}^{\times}$. Here $\mathbb{k}_{a}=\mathbb{k}$ with $y \cdot 1=0, g \cdot 1=a$; and $\mathcal{U}_{a, b}=\mathbb{k}^{n}$ with $g \cdot e_{i}=a q^{i-1} e_{i}, i \in \mathbb{I}_{n}, y \cdot e_{j}=e_{j+1}, j \in \mathbb{I}_{n-1}, y \cdot e_{n}=b e_{1}$.

2.2. Indecomposable modules. Let $V \in \operatorname{rep} L, \operatorname{dim} V=n \in \mathbb{N}$. Since $g^{2}$ is central, $V[\lambda]:=V_{g^{2}}^{(\lambda)}=V_{g}^{(a)} \oplus V_{g}^{(-a)} \leq V$ for any $\lambda=a^{2} \in \mathbb{k}^{\times}$. Let $\operatorname{rep}^{\lambda} L$ be the full subcategory of rep $L$ of those $V$ such that $V=V[\lambda]$.

Lemma 2.3. (i) $\operatorname{rep} L=\oplus_{\lambda \in \mathbb{k}} \times \operatorname{rep}^{\lambda} L$ is a graded tensor category, $i$. e. $\otimes: \operatorname{rep}^{\lambda} L \times \operatorname{rep}^{\mu} L \rightarrow \operatorname{rep}^{\lambda \mu} L$ for $\lambda, \mu \in \mathbb{k}^{\times}, \mathbb{k}_{\varepsilon} \in \operatorname{rep}^{1} L$, the dual of $V \in \operatorname{rep}^{\lambda} L$ belongs to $\operatorname{rep}^{\lambda^{-1}} L$. 
(ii) If $\Gamma \leq \mathbb{k}^{\times}$, then $\operatorname{rep}^{\Gamma} L:=\oplus_{\lambda \in \Gamma} \operatorname{rep}^{\lambda} L$ is a graded tensor subcategory of $\operatorname{rep} L$.

(iii) Let $\operatorname{rep}_{1}^{\lambda} L$ be the full subcategory of $\operatorname{rep}^{\lambda} L$ consisting of modules with semisimple action of $g^{2}$. Then $\operatorname{rep}_{1}^{\Gamma} L:=\oplus_{\lambda \in \Gamma} \operatorname{rep}_{1}^{\lambda} L$ is a graded tensor subcategory of rep $L$ for every $\Gamma \leq \mathbb{k}^{\times}$.

(iv) $\operatorname{rep} L^{(1)}$ can be identified with the tensor subcategory $\operatorname{rep}_{1}^{1} L$. More generally, $\operatorname{rep} L^{(n)}$ can be identified with $\operatorname{rep}_{1}^{\mathbb{G}_{n}} L$.

(v) If $\lambda, b \in \mathbb{k}^{\times}$, then $\mathfrak{L}_{b}, \mathfrak{R}_{b}: \operatorname{rep}^{\lambda} L \rightarrow \operatorname{rep}^{\lambda b^{2}} L, \mathfrak{L}_{b}(V)=\mathbb{k}_{b} \otimes V$, $\mathfrak{R}_{b}(V)=V \otimes \mathbb{k}_{b}$ are equivalences of abelian categories; in particular $\mathfrak{L}_{-1}$ and $\mathfrak{R}_{-1}$ are auto-equivalences of $\operatorname{rep}^{\lambda} L$ for all $\lambda$.

(vi) If $V \in \operatorname{rep} L$ is indecomposable, then $V \in \operatorname{rep}^{\lambda} L$ for a unique $\lambda$.

Proof. Let $\operatorname{rep}_{n}^{\lambda} L$ be the full subcategory of $\operatorname{rep} L$ of those $V$ such that $V=\operatorname{ker}\left(g^{2}-\lambda\right)^{n}, n \in \mathbb{N}_{0}$. We claim that $V \otimes W \in \operatorname{rep}_{n m}^{\lambda \mu} L$, whenever $V \in \operatorname{rep}_{n}^{\lambda} L$ and $W \in \operatorname{rep}_{m}^{\mu} L$. The claim follows by induction on $\operatorname{dim} V$ and implies (i). The rest of the proof is standard.

Remark 2.4. The various tensor categories $\operatorname{rep}^{\Gamma} L$ can be realized as the categories of comodules of a suitable Hopf algebra, namely the Hopf subalgebra of the restricted dual of $L$ spanned by the matrix coefficients of the objects in $\operatorname{rep}^{\Gamma} L$. Similarly for variations as in the Lemma.

Remark 2.5. Since $\mathbb{k}_{a}, \mathcal{U}_{a, b} \in \operatorname{rep}^{\lambda} L$ for any $\lambda=a^{2} \in \mathbb{k}^{\times}$, we see that

$$
\operatorname{Ext}_{L}^{1}\left(\mathbb{k}_{a}, \mathbb{k}_{c}\right)=\operatorname{Ext}_{L}^{1}\left(\mathbb{k}_{a}, \mathcal{U}_{c, d}\right)=\operatorname{Ext}_{L}^{1}\left(\mathcal{U}_{a, b}, \mathcal{U}_{c, d}\right)=0,
$$

whenever $a^{2} \neq c^{2}$.

2.2.1. Representations with $y=0$. Observe that $L / L y L \simeq \mathbb{k} \mathbb{Z}$. Thus there is a unique indecomposable module $\mathcal{V}_{a}^{n}$ of dimension $n$ where $y$ acts by 0 , namely with $g$ acting by $\mathrm{J}_{n}(a)$ with $a \in \mathbb{k}^{\times}$. Given $n, m \in \mathbb{N}$ and $a \in \mathbb{k}^{\times}$, it is easy to see that $\mathcal{V}_{a}^{n} \simeq \mathbb{k}_{a} \otimes \mathcal{V}_{1}^{n}, \mathcal{V}_{1}^{n} \otimes \mathcal{V}_{1}^{m} \simeq \mathcal{V}_{1}^{m} \otimes \mathcal{V}_{1}^{n}$. Let $n, m \in \mathbb{N}$, $2 \leq m \leq n$. By [․, Corollary 1], we have

$$
\mathcal{V}_{1}^{n} \otimes \mathcal{V}_{1}^{m} \simeq \oplus_{k=1}^{m} \mathcal{V}_{1}^{n-(m-(2 k-1))} .
$$

2.2.2. Representations with $y \neq 0$. We focus next on indecomposable $L$ modules with $y \neq 0$. Since $g$ has a unique eigenvalue $a$ if and only if $y$ acts by $0, g$ has eigenvalues $\pm a$. It would be enough to assume that $V \in \operatorname{rep}^{1} L$ since the indecomposable modules in $\operatorname{rep}^{\lambda} L$ can be deduced by Lemma 2.3 (v) but the analysis is the same as in the general case. So assume that $V \in \operatorname{rep}^{\lambda} L$ indecomposable, $\lambda=a^{2}$. Let $\mathfrak{B}_{ \pm}$be a basis of $V_{g}^{( \pm a)}$ and $\mathfrak{B}:=\mathfrak{B}_{+} \cup \mathfrak{B}_{-} ;$let $\ell=\operatorname{dim} V_{g}^{(a)}, \wp=n-\ell$. Up to replacing $V$ by $\mathfrak{L}_{-1}(V)$ (i. e. interchanging $a$ and $-a$ ), we may, and always will, assume that $\ell \geq \wp$. Then

$$
[g]_{\mathfrak{B}}=\left(\begin{array}{cc}
A & 0 \\
0 & B
\end{array}\right), \quad[y]_{\mathfrak{B}}=\left(\begin{array}{cc}
0 & C \\
D & 0
\end{array}\right)
$$


where $A \in \mathbf{G L}_{\ell}(\mathbb{k}), B \in \mathbf{G L}_{\wp}(\mathbb{k}), C \in \mathrm{M}_{\ell \times \wp}(\mathbb{k})$ and $D \in \mathrm{M}_{\wp \times \ell}(\mathbb{k})$. Conversely, $g$ and $y$ given by (2.3) define a representation of $L$ if and only if

$$
A C=-C B, \quad B D=-D A .
$$

We fix a pair of matrices $A$ and $B$ in Jordan form. Our goals are to describe conditions on $y$ equivalent to the indecomposability of $V$ and then to classify the pairs $(C, D)$ satisfying these conditions. In general, let

$$
\begin{aligned}
& \mathbb{H}=\mathbb{H}_{A, B}=\left\{\left(t_{1}, t_{2}\right) \in \mathbf{G L}_{\ell}(\mathbb{k}) \times \mathbf{G L}_{\wp}(\mathbb{k}): t_{1} A=A t_{1}, t_{2} B=B t_{2}\right\}, \\
& \mathfrak{V}=\mathfrak{V}_{A, B}=\left\{(C, D) \in \mathrm{M}_{\ell \times \wp}(\mathbb{k}) \times \mathrm{M}_{\wp \times \ell}(\mathbb{k}): \text { (2.4) holds and } V \text { is simple }\right\} .
\end{aligned}
$$

Then the algebraic group $\mathbb{H}$ acts on $\mathfrak{V}$ and our goals can be rephrased as:

- Describe $\mathfrak{V}$ and decide when is non-empty,

o determine the orbits of the action of $\mathbb{H}$ on $\mathfrak{V}$.

We approach these questions by elementary means when $A$ and $B$ have simple Jordan forms and apply the obtained results to the classification of the indecomposable modules of dimension $\leq 5$.

In this section, we set $U_{g}^{( \pm a)}=U^{( \pm a)}$ and $y_{ \pm a}=y_{\mid U^{( \pm a)}}$ for any $U \in \operatorname{rep} L$.

2.2.3. Representations with $g$ semisimple. Here $\operatorname{dim} V=n \geq 3$ and $g$ acts by $\left(\begin{array}{cc}a \operatorname{id}_{\ell} & 0 \\ 0 & -a \operatorname{id}_{\wp}\end{array}\right)$ where $0<\ell<n$ and $\wp=n-\ell$; any $C, D$ satisfy (2.4).

Lemma 2.6. If either $y_{a}=0$ or $y_{-a}=0$, then $V$ is decomposable.

Proof. This is clear if $y_{a}=0$ and $y_{-a}=0$. If $y_{a}=0$ and $y_{-a} \neq 0$, then take a basis $v_{1}, \ldots, v_{q}$ of $\operatorname{Im}\left(y_{-a}\right)$, say $v_{j}=y_{-a}\left(v_{\ell+j}\right), j \in \mathbb{I}_{q}$, and a basis $v_{\ell+q+1}, \ldots, v_{\wp}$ of ker $y_{-a}$. Complete to a basis $v_{1}, \ldots, v_{q}, \ldots, v_{\ell}$ of $V^{a}$. Then $V=\left\langle v_{1}, v_{\ell+1}\right\rangle \oplus\left\langle v_{2}, \ldots, v_{\ell}, v_{\ell+2}, \ldots, v_{\wp}\right\rangle$ is decomposable. If $y_{a} \neq 0$ and $y_{-a}=0$, then $\mathfrak{L}_{-1}(V)$ is decomposable by the preceding, and so is $V$.

Assume that $\ell=n-1$. Define the representation $\mathcal{C}_{a}^{n}$ by $y \mapsto\left(\begin{array}{cc}0 & e_{1} \\ e_{\ell}^{t} & 0\end{array}\right)$.

Lemma 2.7. Assume that $\ell=n-1, y_{a} \neq 0$ and $y_{-a} \neq 0$.

(i) If $y_{a} y_{-a} \neq 0$, then $V$ is decomposable.

(ii) $y_{a} y_{-a}=0$ if and only if $V \simeq \mathcal{C}_{a}^{n}$.

(iii) $\mathcal{C}_{a}^{n}$ is indecomposable if and only if $n=3$.

Proof. (i) Assume that $y_{a} y_{-a} \neq 0$. Let $\left(v_{\ell+1}\right)$ be a basis of $V^{-a}$ and let $v_{2}, \ldots, v_{\ell}$ be a basis of ker $y_{a}$. By hypothesis $v_{1}:=y_{-a}\left(v_{\ell+1}\right) \notin$ ker $y_{a}$, hence $v_{1}, \ldots, v_{\ell}$ is a basis of $V^{a}$. Then $V=\left\langle v_{1}, v_{\ell+1}\right\rangle \oplus\left\langle v_{2}, \ldots, v_{\ell}\right\rangle$ is decomposable. (ii) Since $n>2, y_{a} y_{-a}=0$ in $\mathcal{C}_{a}^{n}$. Assume that $y_{a} y_{-a}=0$. Let $\left(v_{\ell+1}\right)$ be a basis of $V^{-a}$ and $v_{1}:=y_{-a}\left(v_{\ell+1}\right) \in \operatorname{ker} y_{a}$; complete to a basis $v_{1}, \ldots, v_{\ell-1}$ of ker $y_{a}$. Let $v_{\ell} \in V^{a}$ be such that $y_{a}\left(v_{\ell}\right)=v_{\ell+1}$. Then $v_{1}, \ldots, v_{\ell+1}$ is a basis of $V$ that provides the isomorphism with $\mathcal{C}_{a}^{n}$. 
(iii) If $n>3$, then $\left\langle e_{1}, e_{\ell}, e_{\ell+1}\right\rangle \oplus\left\langle e_{2}, \ldots, e_{\ell-1}\right\rangle$ is a decomposition of $V=$ $\mathcal{C}_{a}^{n}$. Assume that $n=3$ and that $V=U \oplus W$ is decomposable. Hence $V^{ \pm a}=U^{ \pm a} \oplus W^{ \pm a}$ so that $U^{-a}=0$ and $V^{-a}=W^{-a}=\mathbb{k} e_{3}$ or vice versa. If $U^{-a}=0$, then $U^{a} \leq \operatorname{ker} y_{a}=\mathbb{k} e_{1}=\mathbb{k} y_{-a}\left(e_{3}\right) \leq W^{a}$; hence $U=0$.

Assume that $\ell=n-2$. We define the representations $\mathcal{D}_{1, a}^{4}, \mathcal{D}_{2, a, b}^{4}, b \in \mathbb{k}^{\times}$, $\mathcal{D}_{3, a}^{4}$ and $\mathcal{D}_{4, a}^{5}$ by $y \mapsto\left(\begin{array}{cc}0 & C \\ D & 0\end{array}\right)$, where

$\mathcal{D}_{1, a}^{4}: \quad C=\left(\begin{array}{ll}0 & e_{1}\end{array}\right), \quad D=\left(\begin{array}{c}e_{1}^{t} \\ e_{2}^{t}\end{array}\right) ; \quad \mathcal{D}_{2, a, b}^{4}: \quad C=\left(\begin{array}{ll}e_{1} & e_{2}\end{array}\right), \quad D=\left(\begin{array}{c}b e_{1}^{t}+e_{2}^{t} \\ b e_{2}^{t}\end{array}\right) ;$

$\mathcal{D}_{3, a}^{4}: \quad C=\left(\begin{array}{ll}e_{1} & e_{2}\end{array}\right), \quad D=\left(\begin{array}{c}e_{2}^{t} \\ 0\end{array}\right) ; \quad \mathcal{D}_{4, a}^{5}: \quad C=\left(\begin{array}{ll}e_{1} & e_{2}\end{array}\right), \quad D=\left(\begin{array}{c}e_{2}^{t} \\ e_{3}^{t}\end{array}\right)$.

Lemma 2.8. Let $V \in \operatorname{rep} L$ such that $n \geq 4$ and $g$ acts by $\left(\begin{array}{cc}a \mathrm{id}_{\ell} & 0 \\ 0 & -a \mathrm{id}_{2}\end{array}\right)$.

(i) If $n=4$, then $V$ is indecomposable iff $V \simeq \mathcal{D}_{1, a}^{4}$, or $\mathcal{D}_{2, a, b}^{4}$ or $\mathcal{D}_{3, a}^{4}$.

(ii) If $n=5$, then $V$ is indecomposable iff $V \simeq \mathcal{D}_{4, a}^{5}$.

(iii) If $n \geq 6$, then $V$ is decomposable.

Proof.

Step 1. The $L$-modules $\mathcal{D}_{1, a}^{4}, \mathcal{D}_{2, a, b}^{4}, \mathcal{D}_{3, a}^{4}$ and $\mathcal{D}_{4, a}^{5}$ are indecomposable.

Suppose that $V=\mathcal{D}_{1, a}^{4}$ is decomposable, i. e. there exist non-zero $L$ submodules $U$ and $W$ such that $V=W \oplus U$. Then $V^{-a}=W^{-a} \oplus U^{-a}$. If $W^{-a}=0$, then $y W^{a}=0$, hence $W^{a}=0$ since $y_{a}$ is injective. Thus $\operatorname{dim} W^{-a}=\operatorname{dim} U^{-a}=1$. If $0 \neq w=\alpha e_{3}+\beta e_{4} \in W^{-a}$, then $y^{2}(w)=$ $\beta e_{3} \in W^{-a}$, so that either $\alpha=0$ or $\beta=0$, and similarly for $U^{-a}$. In other words, we may assume that $W^{-a}=\left\langle e_{3}\right\rangle$ and $U^{-a}=\left\langle e_{4}\right\rangle$, but $y^{2}\left(e_{4}\right)=e_{3} \in$ $W^{-a} \cap U^{-a}$, a contradiction. By a similar argument, $\mathcal{D}_{4, a}^{5}$ is indecomposable. Clearly $\mathcal{D}_{3, a}^{4} \simeq\left(\mathcal{D}_{1, a^{-1}}^{4}\right)^{*}$, consequently it is indecomposable.

Finally, suppose that $\mathcal{D}_{2, a, b}^{4}=U \oplus W$ for some $L$-submodules $U$ and $W$. Pick a square root $\sqrt{b}$ of $b$ and note that $\lambda_{i}=(-1)^{i} \sqrt{b}, i=1,2$, are the eigenvalues of $y$ and $V_{y}^{\lambda_{i}}=\left\langle v_{i}:=e_{1}+\lambda_{i} e_{3}\right\rangle$ are the respective eigenspaces. If $v_{1}=u+w$ with $u \in U$ and $w \in W$, then $u, w \in V_{y}^{\lambda_{1}}$. If $u \neq 0$ and $w \neq 0$, then $v_{1} \in U \cap W=0$, a contradiction. If $w=0$, then $v_{1} \in U$ and $v_{2}=g v_{1}=g u \in U$ which implies $W=0$. Similarly, $u=0$ implies $U=0$.

Step 2. If $n \geq 5$ and $y_{a} y_{-a}$ is an isomorphism, then $V$ is decomposable.

Note that $y_{a} y_{-a}$ is an isomorphism if and only if $y_{-a}$ is injective, $y_{a}$ is surjective and $\operatorname{Im} y_{-a} \cap \operatorname{ker} y_{a}=0$. Let $e_{\ell+1}, e_{\ell+2}$ be a basis of $V^{-a}=\operatorname{Im} y_{a}$. Denote by $e_{i}=y_{-a}\left(e_{i+2}\right) \neq 0$, where $i \in \mathbb{I}_{\ell-1, \ell}$. So $e_{i} \notin \operatorname{ker} y_{a}$. We complete to a basis $e_{1}, \cdots, e_{\ell}$ of $V^{a}$ such that $y_{a}\left(e_{j}\right)=0$, for all $j \in \mathbb{I}_{1, \ell-2}$. Therefore $V=\left\langle e_{1}, \cdots, e_{\ell-2}\right\rangle \oplus\left\langle e_{\ell-1}, e_{\ell}, e_{\ell+1}, e_{\ell+2}\right\rangle$ is decomposable.

We next investigate what happens when $y_{a} y_{-a}$ is not an isomorphism. 
Step 3. Assume that $y_{-a}$ is injective and $\operatorname{Im} y_{-a} \cap \operatorname{ker} y_{a} \neq 0$.

(a) If $n \geq 6$ and $y_{a}$ is surjective then $V$ is decomposable.

(b) If $n \geq 5$ and $y_{a}$ is not surjective then $V$ is decomposable.

Let $0 \neq e_{1} \in \operatorname{Im} y_{-a} \cap \operatorname{ker} y_{a}$. Pick $e_{\ell+1} \in V^{-a}$ such that $e_{1}=y_{-a}\left(e_{\ell+1}\right)$ and complete to a basis $e_{\ell+1}, e_{\ell+2}$ of $V^{-a}$. Let $e_{2}:=y_{-a}\left(e_{\ell+2}\right)$.

(a) If $e_{2} \in \operatorname{ker} y_{a}$, then complete to a basis $e_{1}, \ldots, e_{\ell-2}$ of ker $y_{a}$. Take $e_{\ell-1}, e_{\ell} \in V^{a}$ such that $y_{a}\left(e_{\ell-1}\right)=e_{\ell+1}$ and $y_{a}\left(e_{\ell}\right)=e_{\ell+2}$. Clearly $e_{1}, \ldots, e_{\ell}$ is a basis of $V^{a}$. Since $\ell \geq 4, V=\left\langle e_{1}, e_{\ell-1}, e_{\ell+1}\right\rangle \oplus\left\langle e_{2}, \cdots, e_{\ell-2}, e_{\ell}, e_{\ell+2}\right\rangle$ is decomposable. If $e_{2} \notin \operatorname{ker} y_{a}$, then consider $e_{1}, e_{3}, \cdots, e_{\ell-1}$ a basis of ker $y_{a}$. We can find $e_{\ell} \in V^{a}$ such that $e_{1}, e_{3}, \cdots, e_{\ell-1}, e_{2}, e_{\ell}$ is a basis of $V^{a}$. Since $\ell \geq 4, V=\left\langle e_{1}, e_{2}, e_{\ell}, e_{\ell+1}, e_{\ell+2}\right\rangle \oplus\left\langle e_{3}, \cdots, e_{\ell-1}\right\rangle$ is decomposable.

(b) If $e_{2} \in$ ker $y_{a}$, then we complete to a basis $e_{1}, e_{2} \cdots, e_{\ell-1}$ of ker $y_{a}$ and then to a basis $e_{1}, \cdots, e_{\ell}$ of $V^{a}$. So $y_{a}\left(e_{\ell}\right)=b e_{\ell+1}+c e_{\ell+2}, b, c \in \mathbb{k}, b c \neq 0$. Then either $V=\left\langle b e_{1}+c e_{2}, e_{\ell}, b e_{\ell+1}+c e_{\ell+2}\right\rangle \oplus\left\langle e_{2}, \cdots, e_{\ell-1}, e_{\ell+2}\right\rangle$ if $b \in \mathbb{k}^{\times}$, or else $V=\left\langle e_{1}, e_{3}, \cdots, e_{\ell-1}, e_{\ell+1}\right\rangle \oplus\left\langle e_{2}, e_{\ell}, e_{\ell+2}\right\rangle$ if $b=0$ and $c \in \mathbb{k}^{\times}$. That is, $V$ is decomposable. If $e_{2} \notin \operatorname{ker} y_{a}$, take a basis $e_{1}, e_{3}, \cdots, e_{\ell}$ of ker $y_{a}$, so that $e_{1}, e_{2}, \cdots, e_{\ell}$ is a basis of $V^{a}$. Then $V=\left\langle e_{1}, e_{2}, e_{\ell+1}, e_{\ell+2}\right\rangle \oplus\left\langle e_{3}, \cdots, e_{\ell}\right\rangle$ is decomposable.

Step 4. If $n \geq 4, y_{-a}$ is not injective and $\operatorname{Im} y_{-a} \cap \operatorname{ker} y_{a} \neq 0$, then $V$ is decomposable.

Let $0 \neq e_{1} \in \operatorname{Im} y_{-a} \cap \operatorname{ker} y_{a}$. Pick $e_{\ell+2} \in V^{-a}$ such that $e_{1}=y_{-a}\left(e_{\ell+2}\right)$ and complete to a basis $e_{\ell+1}, e_{\ell+2}$ of $V^{-a}$ with $y_{-a}\left(e_{\ell+1}\right)=0$. If $y_{a}$ is surjective, take $e_{\ell+1}=y_{a}\left(e_{\ell-1}\right)$ and $e_{\ell+2}=y_{a}\left(e_{\ell}\right)$ and complete to a basis $e_{1}, e_{2}, \ldots, e_{\ell}$ of $V^{a}$ such that $e_{i} \in \operatorname{ker} y_{a}, i \in \mathbb{I}_{\ell-2}$. Then $V=$ $\left\langle e_{1}, e_{\ell}, e_{\ell+2}\right\rangle \oplus\left\langle e_{2}, \ldots, e_{\ell-1}, e_{\ell+1}\right\rangle$ is decomposable. If $y_{a}$ is not surjective, then take $e_{1}, e_{2} \ldots, e_{\ell-1}$ be a basis of ker $y_{a}$ such that $e_{1}, e_{2} \ldots, e_{\ell}$ is a basis of $V^{a}$. Then $V=\left\langle e_{1}, e_{3}, \ldots, e_{\ell+2}\right\rangle \oplus\left\langle e_{2}\right\rangle$ is decomposable.

Step 5. Assume that $y_{-a}$ is not injective and $\operatorname{Im} y_{-a} \cap \operatorname{ker} y_{a}=0$.

(a) If $n \geq 5$ and $y_{a}$ is surjective then $V$ is decomposable.

(b) If $n \geq 4$ and $y_{a}$ is not surjective then $V$ is decomposable.

(a) Let $e_{\ell+1}, e_{\ell+2}$ be a basis of $V^{-a}$ such that $y_{-a}\left(e_{\ell+1}\right)=0$ and $y_{-a}\left(e_{\ell+2}\right)$ $=e_{\ell} \neq 0$, consequently $e_{\ell} \notin \operatorname{ker} y_{a}$. Thus we can consider $e_{1}, e_{2}, \ldots, e_{\ell}$ a basis of $V^{a}$ such that $e_{1}, e_{2}, \ldots, e_{\ell-2} \in \operatorname{ker} y_{a}$. Therefore $V=\left\langle e_{1}, \ldots, e_{\ell-2}\right\rangle \oplus$ $\left\langle e_{\ell-1}, e_{\ell}, e_{\ell+1}, e_{\ell+2}\right\rangle$ is decomposable. (b) is similar to (a).

Step 6. Proof of the Lemma.

(i) By Steps 2, 3, 4 and 5it is enough to consider the cases:

- $y_{a} y_{-a}$ is an isomorphism.

$\circ y_{-a}$ is injective and $y_{a}$ is not surjective.

- $y_{-a}$ is not injective and $y_{a}$ is surjective.

In the first case we have two possibilities for the canonical Jordan form of $y_{a} y_{-a}$, namely diagonal or $J_{2}(b)$, with $b \in \mathbb{k}^{\times}$. Consider $e_{3}, e_{4}$ basis of $V^{-a}$ 
such that the canonical Jordan form of $y_{a} y_{-a}$ is diagonal. Take $e_{1}=y\left(e_{3}\right)$ and $e_{2}=y\left(e_{4}\right)$. Then $V=\left\langle e_{1}, e_{3}\right\rangle \oplus\left\langle e_{2}, e_{4}\right\rangle$ is decomposable. Now if the canonical Jordan form of $y_{a} y_{-a}$ is $J_{2}(b)$ then $V=\mathcal{D}_{2, a, b}^{4}$.

In the second case, if $y_{a} y_{-a}$ is diagonalizable then $V$ is decomposable and otherwise $V=\mathcal{D}_{3, a}^{4}$.

Finally in the third case, consider $e_{3} \in \operatorname{ker} y_{-a}$ and complete to a basis $e_{3}, e_{4}$ of $V^{-a}$ such that $y_{a} y_{-a}\left(e_{4}\right)=b e_{4}, b \in \mathbb{k}^{\times}$. Take $e_{1}=y_{-a}\left(e_{4}\right)$ and complete to a basis $e_{1}, e_{2}$ of $V^{a}$ with $y_{a}\left(e_{2}\right)=c e_{3}+d e_{4}, c \in \mathbb{k}^{\times}$. So $V=\left\langle e_{1}, e_{4}\right\rangle \oplus\left\langle-d b^{-1} e_{1}+e_{2}, e_{3}\right\rangle$ is decomposable. Consider $e_{3}, e_{4}$ a basis of $V^{-a}$ such that the canonical Jordan form of $y_{a} y_{-a}$ is $J_{2}(0)$. Take $e_{1}=y_{a}\left(e_{4}\right)$, $e_{1}, e_{2}$ a basis of $V^{a}$ with $y_{a}\left(e_{2}\right)=b e_{3}+c e_{4}, c \in \mathbb{k}^{\times}$. Taking the basis $e_{1}, c^{-1} e_{2}, e_{3}, b c^{-1} e_{3}+e_{4}$ we have that $V \simeq \mathcal{D}_{1, a}^{4}$.

(ii) By Steps 2, 3, 4 and 5 it is enough to consider the case where $y_{-a}$ is injective, $y_{a}$ is surjective and $\operatorname{Im} y_{-a} \cap \operatorname{ker} y_{a} \neq 0$. Let $0 \neq e_{1} \in$ $\operatorname{Im} y_{-a} \cap \operatorname{ker} y_{a}$. Pick $e_{4} \in V^{-a}$ such that $e_{1}=y_{-a}\left(e_{4}\right)$ and complete to a basis $e_{4}, e_{5}$ of $V^{-a}$. Denote by $e_{2}=y_{-a}\left(e_{5}\right)$. Let $y e_{2}=\alpha e_{4}+\beta e_{5}$, $y e_{3}=\gamma e_{4}+\eta e_{5}, A=\left(\begin{array}{cc}\alpha & \gamma \\ \beta & \eta\end{array}\right)$. Taking the basis $e_{1}, \eta(\operatorname{det} A)^{-1} e_{2}-$ $\beta(\operatorname{det} A)^{-1} e_{3},-\gamma(\operatorname{det} A)^{-1} e_{2}+\alpha(\operatorname{det} A)^{-1} e_{3}, e_{4}, e_{5}$, we have that $V \simeq \mathcal{D}_{4, a}^{5}$. (iii) follows from the Steps 2, 3, 4 and 5.

\subsubsection{Representations with A a Jordan block.}

Lemma 2.9. Let $V$ be a representation of $L$ given by (2.3) where $A$ and $B$ are Jordan blocks. Then $V$ is indecomposable if and only if $y \neq 0$.

Proof. Let $V=U \oplus W$ be a decomposition with both $U$ and $W$ not 0 . Hence $V^{( \pm a)}=U^{( \pm a)} \oplus W^{( \pm a)}$. Since $A$ is a Jordan block, either $U^{(a)}=0$ and $W^{(a)}=V^{(a)}$ or vice versa. If $U^{(a)}=0$, then $U^{(-a)}=V^{(-a)}$ and $W^{(-a)}=0$, because $B$ is also a Jordan block; thus $y_{-a}=y U^{(-a)} \leq U^{(a)}=0$ and similarly $y\left(V^{(a)}\right)=0$, implying $y=0$. Same if $U^{(a)}=V^{(a)}$. The converse is evident.

Assume that $n \geq 3$ and $\ell=n-1$. Define the representations $\mathcal{E}_{1, a}^{n}, \mathcal{E}_{2, a}^{n}$ and $\mathcal{E}_{3, a, b}^{n}, b \in \mathbb{k}^{\times}$by (2.3), where $A=\mathrm{J}_{\ell}(a), B=-a$, and $y$ acts as follows:

$$
\mathcal{E}_{1, a}^{n}: y \mapsto\left(\begin{array}{cc}
0 & e_{1} \\
0 & 0
\end{array}\right), \quad \mathcal{E}_{2, a}^{n}: y \mapsto\left(\begin{array}{cc}
0 & 0 \\
e_{\ell}^{t} & 0
\end{array}\right), \quad \mathcal{E}_{3, a, b}^{n}: y \mapsto\left(\begin{array}{cc}
0 & b e_{1} \\
e_{\ell}^{t} & 0
\end{array}\right) .
$$

These are pairwise non-isomorphic, for different values of $a$ and $b$.

Lemma 2.10. Let $n \geq 3$ and $\ell=n-1$. Let $V$ be a representation of $L$ given by (2.3) where $A=\mathrm{J}_{\ell}(a)$ and $B=-a$. Then the following are equivalent:

(i) $V$ is indecomposable,

(ii) $y \neq 0$,

(iii) $V$ is isomorphic to one, and only one, of $\mathcal{E}_{1, a}^{n}, \mathcal{E}_{2, a}^{n}$ or $\mathcal{E}_{3, a, b}^{n}$, for unique $a, b \in \mathbb{k}^{\times}$. 
Proof. Here $C=v, D=w^{t}$ where $v, w \in \mathbb{k}^{\ell}$ and (2.4) says that $A v=a v$, $A^{t} w=a w$. Thus $v=b e_{1}, w=c e_{\ell}$ for some $b, c \in \mathbb{k}$, and $V$ is indecomposable iff $(b, c) \neq 0$ by Lemma2.9. If $c=0$, then the basis $b e_{1}, \ldots, e_{n}$ gives $V \simeq \mathcal{E}_{1, a}^{n}$. If $c \neq 0$, then $e_{1}, \ldots, c e_{n}$ gives $V \simeq \mathcal{E}_{2, a}^{n}$ when $b=0$, or $\mathcal{E}_{3, a, b c}^{n}$ when $b \neq 0$.

Assume that $n \geq 4$ and $\ell=n-2$, so that $A=\mathrm{J}_{\ell}(a)$ and $B=\mathrm{J}_{2}(-a)$. Consider $C_{1}, C_{2} \in \mathrm{M}_{\ell \times \wp}(\mathbb{k})$ and $D, D_{i} \in \mathrm{M}_{\wp \times \ell}(\mathbb{k})$, given by:

$C_{1}=\left(\begin{array}{ll}0 & e_{1}\end{array}\right), \quad C_{2}=\left(\begin{array}{ll}e_{1} & -e_{2}\end{array}\right), \quad D=\left(\begin{array}{c}e_{l}^{t} \\ 0\end{array}\right), \quad D_{i}=\left(\begin{array}{c}e_{i}^{t} \\ -e_{i+1}^{t}\end{array}\right), \quad i \in \mathbb{I}_{1, \ell-1}$.

We define the following representations of $L$ on $\mathbb{k}^{n}$ by (2.3) where $y$ acts by:

$$
\begin{array}{ll}
\mathcal{F}_{1, a}^{n}: y \mapsto\left(\begin{array}{cc}
0 & C_{1} \\
0 & 0
\end{array}\right), & \mathcal{F}_{2, a}^{n}: y \mapsto\left(\begin{array}{cc}
0 & C_{2} \\
0 & 0
\end{array}\right), \\
\mathcal{F}_{3, a}^{n}: y \mapsto\left(\begin{array}{cc}
0 & 0 \\
D & 0
\end{array}\right), & \mathcal{F}_{4, a}^{n, i}: y \mapsto\left(\begin{array}{cc}
0 & 0 \\
D_{i} & 0
\end{array}\right), \\
\mathcal{F}_{5, a, b, c}^{n}: y \mapsto\left(\begin{array}{cc}
0 & b C_{1} \\
c D & 0
\end{array}\right), & \mathcal{F}_{6, a, b, c}^{n, i}: y \mapsto\left(\begin{array}{cc}
0 & b C_{1} \\
c D_{i} & 0
\end{array}\right), \\
\mathcal{F}_{7, a, b, c}^{n}: y \mapsto\left(\begin{array}{cc}
0 & b C_{2} \\
c D & 0
\end{array}\right), & \mathcal{F}_{8, a, b, c}^{n, i}: y \mapsto\left(\begin{array}{cc}
0 & b C_{2} \\
c D_{i} & 0
\end{array}\right) .
\end{array}
$$

Lemma 2.11. Let $V$ be a representation of $L$ given by (2.3) where $A=\mathrm{J}_{\ell}(a)$ and $B=\mathrm{J}_{2}(-a)$, with $n \geq 4$. Then the following are equivalent:

(i) $V$ is indecomposable.

(ii) $V$ is isomorphic to one of $\mathcal{F}_{j, a}^{n}, j \in \mathbb{I}_{3}, \mathcal{F}_{4, a}^{n, i}, \mathcal{F}_{j, a, b, c}^{n}, j=5,7$ and $\mathcal{F}_{j, a, b, c}^{n, i}, j=6,8$, for unique $a, b, c \in \mathbb{k}^{\times}$.

If $n \geq 5$, then the above representations are pairwise non-isomorphic. If $n=4$, then $\mathcal{F}_{4, a}^{4,1} \simeq \mathcal{F}_{3,-a}^{4}, \mathcal{F}_{2, a}^{4} \simeq \mathcal{F}_{4,-a}^{4,1}, \mathcal{F}_{7, a, b, c}^{4} \simeq \mathcal{F}_{6,-a, c b}^{4,1}$.

Proof. By Lemma 2.9, we may assume that $y \neq 0$. Then the Lemma follows by a lengthy but straightforward analysis.

Let $n \geq 4, \ell=n-2$ and $\wp=2$. Define the representation $\mathcal{G}_{a}^{n}$ by (2.3), where $A=\mathrm{J}_{\ell}(a), B=-a \operatorname{Id}_{2}, C=\left(0 e_{1}\right)$ and $D=\left(\begin{array}{c}e_{\ell}^{t} \\ 0\end{array}\right)$.

Lemma 2.12. Assume that $\ell, \wp \geq 2$. Let $V$ be a representation of $L$ given by (2.3) where $A=\mathrm{J}_{\ell}(a)$ and $B=-a \operatorname{Id}_{\wp}$. Then the following are equivalent:

(i) $V$ is indecomposable.

(ii) $\wp=2$ and $V \simeq \mathcal{G}_{a}^{n}$.

Proof. Let $C \in \mathrm{M}_{\ell \times \wp}(\mathbb{k})$ and $D \in \mathrm{M}_{\wp \times \ell}(\mathbb{k})$ such that (2.4) holds. By our hypotheses on $A$ and $B$, there exist $c_{1}, \ldots, c_{\wp}, d_{1}, \ldots, d_{\wp} \in \mathbb{k}$ such that

$$
C=\sum_{i \in \mathbb{I}_{\wp}} c_{i} e_{i}, \quad D=\sum_{i \in \mathbb{I}_{\wp}} d_{i} e_{i}^{t} .
$$


Let $y_{ \pm a}=y_{\mid V^{( \pm a)}}$. Since $\operatorname{dim} \operatorname{Im} y_{( \pm a)} \leq 1$ and $\ell, \wp \geq 2$, we see that $\operatorname{ker} y_{( \pm a)} \neq 0$.

Assume first that $y_{a}=0$. Let $U=U^{(a)} \oplus U^{(-a)}$, where $U^{(a)}=0$ and $U^{(-a)}=\operatorname{ker} y_{-a} \neq 0$; let $W=W^{(a)} \oplus W^{(-a)}$, where $W^{(-a)}$ is a direct summand of ker $y_{-a}$ in $V^{(-a)}$ and $W^{(a)}=V^{(a)}$. Then $V=U \oplus W$ is a decomposition in $\operatorname{rep} L$. So, assume that $y_{a} \neq 0$.

○ If $\operatorname{Im} y_{a} \cap \operatorname{ker} y_{-a}=0$, then pick a direct summand $Z$ of $\operatorname{Im} y_{a} \oplus \operatorname{ker} y_{-a}$ in $V^{(-a)}$ and set $U=U^{(-a)}=\operatorname{ker} y_{-a}, W=V^{(a)} \oplus\left(\operatorname{Im} y_{a} \oplus Z\right)$.

- If $\operatorname{Im} y_{a} \lesseqgtr \operatorname{ker} y_{-a}$, then pick a direct summand $Z_{1}$ of $\operatorname{Im} y_{a}$ in ker $y_{-a}$ and a direct summand $Z_{2}$ of ker $y_{-a}$ in $V^{(-a)}$. Then set $U=U^{(-a)}=Z_{1} \neq 0$, $W=V^{(a)} \oplus\left(\operatorname{Im} y_{a} \oplus Z_{2}\right)$.

Hence $V=U \oplus W$ is a decomposition in $\operatorname{rep} L$ in both cases.

It remains the case $\operatorname{Im} y_{a}=\operatorname{ker} y_{-a}$, necessarily $\wp=2$ and $y_{-a} \neq 0$. Let $v_{1}, v_{2} \in \mathbb{k}^{2}$ be such that $\operatorname{Im} y_{a}=\mathbb{k} v_{1}$ and $y_{-a}\left(v_{2}\right)=e_{1}$. Then $\mathbb{k}^{2}=\mathbb{k} v_{1} \oplus \mathbb{k} v_{2}$ and considering the basis $e_{1}, \ldots, e: \ell, v_{1}, v_{2}$, we see that $V \simeq \mathcal{G}_{a}^{n}$.

Finally, we show that $V=\mathcal{G}_{a}^{n}$ is indecomposable. Indeed, let $V=U \oplus W$ be a decomposition in $\operatorname{rep} L$. Then $V^{(a)}=U^{(a)} \oplus W^{(a)}$ and either $U^{(a)}=0$ or $W^{(a)}=0$; suppose the first happens. Then $U^{(-a)} \leq \operatorname{ker} y_{-a}=y_{a}\left(V^{(a)}\right)=$ $y_{a}\left(W^{(a)}\right) \leq W^{(-a)}$, hence $U^{(-a)}=0$ and a fortiori $U=0$.

2.2.5. Representations with A sum of a Jordan block and a line. Assume $n \geq 4$. We fix $A=\left(\begin{array}{cc}\mathrm{J}_{n-2}(a) & \\ & a\end{array}\right), B=-a$. Define the representations $\mathcal{H}_{1, a}^{n}, \mathcal{H}_{2, a}^{n}, \mathcal{H}_{3, a, b}^{n}, b \in \mathbb{k}^{\times}$by (2.3), where $y$ acts on $\mathbb{k}^{n}$ by:

$\mathcal{H}_{1, a}^{n}: y \mapsto\left(\begin{array}{cc}0 & e_{1} \\ e_{n-1}^{t} & 0\end{array}\right), \mathcal{H}_{2, a}^{n}: y \mapsto\left(\begin{array}{cc}0 & e_{n-1} \\ e_{n-2}^{t} & 0\end{array}\right), \mathcal{H}_{3, a, b}^{n}: y \mapsto\left(\begin{array}{cc}0 & e_{n-1} \\ b e_{n-1}^{t} & 0\end{array}\right)$.

It is easy to see that these modules are indecomposable.

Lemma 2.13. Let $V$ be a representation of $L$ on $\mathbb{k}^{n}$ given by (2.3), with $A, B$ as above. Then the following statements are equivalent:

(i) $V$ is indecomposable.

(ii) $V$ is isomorphic to exactly one of $\mathcal{H}_{1, a}^{n}, \mathcal{H}_{2, a}^{n}$ or $\mathcal{H}_{3, a, b}^{n}$, for unique $b \in \mathbb{k}^{\times}$.

Proof.

Step 1. There are $b, c, d, f \in \mathbb{k}$ such that $C=b e_{1}+c e_{n-1}, D=d e_{n-2}^{t}+f e_{n-1}^{t}$.

This follows at once from (2.4).

Step 2. If either $y_{-a}=0$ or $y_{a}=0$ then $V$ is decomposable.

Assume $y_{-a}=0$, i. e. $b=c=0$. If $d=0$, then $\left\langle e_{1}, \ldots, e_{n-2}\right\rangle \oplus$ $\left\langle e_{n-1}, e_{n}\right\rangle$ is a decomposition of $V$. If $f=0$, then $\left\langle e_{1}, \ldots, e_{n-2}, e_{n}\right\rangle \oplus\left\langle e_{n-1}\right\rangle$ is a decomposition of $V$. Also $\left\langle d^{-1} e_{1}, \ldots, d^{-1} e_{n-3}, d^{-1} e_{n-2}-f^{-1} e_{n-1}\right\rangle \oplus$ $\left\langle e_{n-1}, e_{n}\right\rangle$ is a decomposition of $V$ when $d, f \neq 0$. The case $y_{a}=0$ is similar.

Step 3. $V$ is indecomposable iff $(b e, c d) \neq 0$. 
Suppose that $(b e, c d)=0$. If $b=0$, then $c \neq 0$ by Step 2. Thus $d=0$ and $\left\langle e_{1}, \ldots, e_{n-2}\right\rangle \oplus\left\langle e_{n-1}, e_{n}\right\rangle$ is a decomposition of $V$. Similarly, if $e=0$, then $d \neq 0, c=0$ and $\left\langle e_{1}, \ldots, e_{n-2}, e_{n}\right\rangle \oplus\left\langle e_{n-1}\right\rangle$ is a decomposition of $V$.

Conversely, suppose that $V=U \oplus W$ with $U, W$ non-trivial submodules of $V$. From $V^{(-a)}=U^{(-a)} \oplus W^{(-a)}$, we may assume that $W^{(-a)}=0$ and $U^{(-a)}=V^{(-a)}$. Since $V^{(a)}=U^{(a)} \oplus W^{(a)}$ and $y W^{(a)} \subset W^{(-a)}=0$, there are three possibilities: if $W^{(a)}=\left\langle e_{n-1}\right\rangle$, then $f=0$; if $W^{(a)}=\left\langle e_{1}, \ldots, e_{n-2}\right\rangle$, then $d=0$; if $W^{(a)}=V^{(a)}$, then $d=f=0$.

Step 4. If $V$ is indecomposable, then it is one of $\mathcal{H}_{1, a}^{n}, \mathcal{H}_{2, a}^{n}$ or $\mathcal{H}_{3, a, b}^{n}$.

By Step 3, $(b f, c d) \neq 0$ and we proceed by a straightforward analysis.

2.2.6. Representations with $A$ a sum of Jordan blocks of the same size. Let $r, t \geq 2$ such that $\ell=r t$ and $\wp \geq 1$. Assume that $A$ consists of $t$ blocks of size $r$, i e. $A=\left(\begin{array}{ccc}\mathrm{J}_{r}(a) & & \\ & \ddots & \\ & & \mathrm{J}_{r}(a)\end{array}\right)$. Let $y_{ \pm a}=y_{\left.\mid V^{( \pm a}\right)}$.

Assume that $t=2$, thus $\ell=2 r$ and $\wp=1$. Define the representation $\mathcal{I}_{a}^{n}$ by $y \mapsto\left(\begin{array}{cc}0 & e_{1} \\ e_{2 r}^{t} & 0\end{array}\right)$.

Lemma 2.14. Assume that $\wp=1$. Let $V$ be a representation of $L$ given by (2.3) where $A$ is as above and $B=-a$. Then the following are equivalent:

(i) $V$ is indecomposable,

(ii) $y_{a} \neq 0, y_{-a} \neq 0, y_{a} y_{-a}=0$ and $t=2$,

(iii) $V \simeq \mathcal{I}_{a}^{n}$.

Proof. Let $C \in \mathrm{M}_{\ell \times \wp}(\mathbb{k})$ and $D \in \mathrm{M}_{\wp \times \ell}(\mathbb{k})$ such that (2.4) holds. By our hypotheses on $A$ and $B$, there exist $c_{1}, \ldots, c_{t}, d_{1}, \ldots, d_{t} \in \mathbb{k}$ such that

$$
C=\sum_{j \in \mathbb{I}_{t}} c_{j} e_{(j-1) r+1}, \quad D=\sum_{j \in \mathbb{I}_{t}} d_{j} e_{j r}^{t} .
$$

We summarize some well-known facts about the $g$-submodules of $V^{a}$ :

(a) Let $T=\left\langle e_{j r}: j \in \mathbb{I}_{t}\right\rangle$. If $w=\sum_{j \in \mathbb{I}_{t}} \alpha_{j} e_{j r} \in T$, then

$$
\langle w\rangle_{g}=\left\langle\sum_{j \in \mathbb{I}_{t}} \alpha_{j} e_{j r+1-i}: i \in \mathbb{I}_{r}\right\rangle \text {. }
$$

(b) If $T=R \oplus S$, then $V^{(a)}=\langle R\rangle_{g} \oplus\langle S\rangle_{g}$.

Clearly, $V$ is decomposable if $y_{a}=0$ and $y_{-a}=0$. Observe that if $y_{-a} \neq 0$, then $\operatorname{Im} y_{-a}\langle C\rangle \subseteq\left\langle w_{1}\right\rangle_{g}$ where $w_{1}=\sum_{j \in \mathbb{I}_{t}} c_{j} e_{j r}$.

Assume that $y_{a}=0$ and $y_{-a} \neq 0$. Then complete $w_{1}$ to a basis $w_{1}, \ldots, w_{t}$ of $T$. Set $U=\oplus_{j \in \mathbb{I}_{2, t}}\left\langle w_{j}\right\rangle_{g}, W=\left\langle w_{1}\right\rangle_{g} \oplus V^{-a}$. Then $V=U \oplus W$ is a decomposition in $\operatorname{rep} L$.

Assume that $y_{a} \neq 0$ and $y_{-a}=0$. If $w \in T \cap \operatorname{ker} y_{a}$, then $\langle w\rangle_{g} \subseteq \operatorname{ker} y_{a}$. By our present hypothesis, $T \cap \operatorname{ker} y_{a} \neq T$. Pick $w \in T-T \cap \operatorname{ker} y_{a}$ and set $U=\left\langle T \cap \operatorname{ker} y_{a}\right\rangle_{g}, W=\langle w\rangle_{g} \oplus V^{(-a)}$. Then $V=U \oplus W$ is a decomposition. 
Assume that $y_{a} \neq 0$ and $y_{-a} \neq 0$. If $y_{a} y_{-a} \neq 0$, then $w_{1} \notin T \cap \operatorname{ker} y_{a}$. Then $V=\left\langle T \cap \operatorname{ker} y_{a}\right\rangle_{g} \oplus\left(\left\langle w_{1}\right\rangle_{g} \oplus V^{(-a)}\right)$ is a decomposition in rep $L$. If otherwise $y_{a} y_{-a}=0$, then $w_{1} \in T \cap \operatorname{ker} y_{a}$; complete to a basis $w_{1}, \ldots, w_{t-1}$ of $T \cap$ ker $y_{a}$ and pick $w_{t} \in T: y_{a}\left(w_{t}\right)=1$. Take in this case $U=\left\langle w_{2}, \ldots, w_{t-1}\right\rangle_{g}$, $W=\left\langle w_{1}, w_{t}\right\rangle_{g} \oplus V^{(-a)}$. Then $V=U \oplus W$ is a decomposition in rep $L$ unless $t=2$ in which case $U=0$. When $t=2$ then the basis of $V^{(a)}$ obtained from those of $\left\langle w_{1}\right\rangle_{g}$ and $\left\langle w_{2}\right\rangle_{g}$ given by (2.5) realizes the isomorphism with $\mathcal{I}_{a}^{n}$.

Finally we show that $\mathcal{I}_{a}^{n}$ is indecomposable. Let $V=U \oplus W$ be a decomposition. Then $V^{( \pm a)}=U^{( \pm a)} \oplus W^{( \pm a)}$. We may assume that $V^{(-a)}=U^{(-a)}$, $W^{(-a)}=0$. Then $W^{(a)} \subseteq \operatorname{ker} y_{a}=\operatorname{Im} y_{-a} \subseteq U^{(a)}$, hence $W=0$.

2.3. Low dimension. In this subsection we classify those $V \in \operatorname{rep} L$ indecomposable of dimension $n \leq 5$. If $g$ has a unique eigenvalue $a$, then $V \simeq \mathcal{V}_{a}^{n}$. So in all proofs below, $g$ has two eigenvalues $\pm a$ and the representation is given by matrices as in (2.3) satisfying (2.4). Also we assume that $\operatorname{dim} V^{(a)}=\ell \geq \wp=\operatorname{dim} V^{(-a)}$, otherwise apply $\mathfrak{L}_{-1}$.

2.3.1. Dimension 2. Given $a \in \mathbb{k}^{\times}$, let $\mathcal{W}_{a}=\mathbb{k}^{2}$ be the representation given by $g \mapsto\left(\begin{array}{cc}a & 0 \\ 0 & -a\end{array}\right), y \mapsto\left(\begin{array}{ll}0 & 1 \\ 0 & 0\end{array}\right)$. By Lemma 2.9, $\mathcal{W}_{a}$ is indecomposable.

Proposition 2.15. Let $V \in \operatorname{rep} L, \operatorname{dim} V=2$. Then $V$ is indecomposable if and only if it is isomorphic to one, and only one, of $\mathcal{U}_{a, b}$ or to $\mathcal{V}_{a}^{2}$ or to $\mathcal{W}_{a}$.

Proof. As discussed above, we may assume that $V$ has a basis $\mathfrak{B}$ such that $[g]_{\mathfrak{B}}=\left(\begin{array}{cc}a & 0 \\ 0 & -a\end{array}\right)$ and $[y]_{\mathfrak{B}}=\left(\begin{array}{cc}0 & b \\ c & 0\end{array}\right)$ for some $b, c \in \mathbb{k}$. Now (2.4) holds. We have four cases: $b=c=0: V \simeq \mathbb{k}_{a} \oplus \mathbb{k}_{-a} ; c=0 \neq b: V \simeq \mathcal{W}_{a} ; b=0 \neq c$ : $V \simeq \mathcal{W}_{-a} ; b, c \in \mathbb{k}^{\times}: V \simeq \mathcal{U}_{a, b c}$

2.3.2. Dimension 3. Assume that $\operatorname{dim} V=3$ and let $a \in \mathbb{k}^{\times}$.

Proposition 2.16. Let $V \in \operatorname{rep} L, \operatorname{dim} V=3$. Then $V$ is indecomposable if and only if $V$ is isomorphic to one, and only one, of $\mathcal{V}_{a}^{3}, \mathcal{C}_{a}^{3}, \mathcal{E}_{1, a}^{3}, \mathcal{E}_{2, a}^{3}$ or $\mathcal{E}_{3, a, b}^{3}$, for unique $a, b \in \mathbb{k}^{\times}$.

Proof. If the action of $g$ is semisimple, then $V \simeq \mathcal{C}_{a}^{3}$ by Lemmas 2.6 and 2.7 , Otherwise Lemma 2.10 applies, since $V$ should have a basis $\mathfrak{B}$ where the action of $g$ is given by $[g]_{\mathfrak{B}}=\left(\begin{array}{cc}\mathrm{J}_{2}(a) & 0 \\ 0 & -a\end{array}\right)$.

2.3.3. Dimension 4. Assume that $\operatorname{dim} V=4$ and let $a \in \mathbb{k}^{\times}$.

Proposition 2.17. Let $V \in \operatorname{rep} L, \operatorname{dim} V=4$. Then $V$ is indecomposable if and only if $V$ is isomorphic to one, and only one, of $\mathcal{V}_{a}^{4}, \mathcal{D}_{1, a}^{4}, \mathcal{D}_{2, a, b}^{4}, \mathcal{D}_{3, a}^{4}$, $\mathcal{E}_{1, a}^{4}, \mathcal{E}_{2, a}^{4}, \mathcal{E}_{3, a, b}^{4}, \mathcal{F}_{1, a}^{4}, \mathcal{F}_{2, a}^{4}, \mathcal{F}_{5, a, b, c}^{4}, \mathcal{F}_{6, a, b, c}^{4,1}, \mathcal{F}_{8, a, b, c}^{4,1}, \mathcal{G}_{a}^{4}, \mathcal{H}_{1, a}^{4}, \mathcal{H}_{2, a}^{4}$ or $\mathcal{H}_{3, a, b}^{4}$ for unique $a, b, c \in \mathbb{k}^{\times}$. 
Proof.

\begin{tabular}{c|c|c|c}
$A$ & $B$ & Indecomposable & Reference \\
\hline$a \mathrm{Id}_{3}$ & $-a$ & none & Lemma [2.7 \\
\hline$a \mathrm{Id}_{2}$ & $-a \mathrm{Id}_{2}$ & $\mathcal{D}_{1, a}^{4}, \mathcal{D}_{2, a, b}^{4}$ or $\mathcal{D}_{2, a, b}^{4}$ & Lemma [2.8 \\
\hline $\mathrm{J}_{3}(a)$ & $-a$ & $\mathcal{E}_{1, a}^{4}, \mathcal{E}_{2, a}^{4}$ or $\mathcal{E}_{3, a, b}^{4}$ & Lemma [2.10] \\
\hline $\mathrm{J}_{2}(a)$ & $\mathrm{J}_{2}(-a)$ & $\mathcal{F}_{1, a}^{4}, \mathcal{F}_{2, a}^{4}, \mathcal{F}_{5, a, b, c}^{4}, \mathcal{F}_{6, a, b, c}^{4,1}$ or $\mathcal{F}_{8, a, b, c}^{4,1}$ & Lemma [2.11 \\
\hline $\mathrm{J}_{2}(a)$ & $-a \mathrm{Id}_{2}$ & $\mathcal{G}_{a}^{4}$ & Lemma [2.12 \\
\hline$\left(\mathrm{J}_{2}(a)\right.$ & $-a$ & $\mathcal{H}_{1, a}^{4}, \mathcal{H}_{2, a}^{4}$ or $\mathcal{H}_{3, a, b}^{4}$ & Lemma 2.13 \\
\hline
\end{tabular}

2.3.4. Dimension 5. Assume that $\operatorname{dim} V=5$ and let $a \in \mathbb{k}^{\times}$.

Proposition 2.18. Let $V \in \operatorname{rep} L, \operatorname{dim} V=5$. Then $V$ is indecomposable if and only if $V$ is isomorphic to one, and only one, of $\mathcal{V}_{a}^{5}, \mathcal{D}_{4, a}^{5}, \mathcal{E}_{1, a}^{5}, \mathcal{E}_{2, a}^{5}$, $\mathcal{E}_{3, a, b, c}^{5}, \mathcal{F}_{j, a}^{5}, j \in \mathbb{I}_{3}, \mathcal{F}_{4, a}^{5, i}, \mathcal{F}_{j, a, b, c}^{5}, j=5,7, \mathcal{F}_{j, a, b, c}^{5, i}, j=6,8, \mathcal{G}_{a}^{5}, \mathcal{H}_{1, a}^{5}, \mathcal{H}_{2, a}^{5}$, $\mathcal{H}_{3, a, b}^{5}$ or $\mathcal{I}_{a}^{5}$ for unique $a, b, c \in \mathbb{k}^{\times}$.

Proof.

\begin{tabular}{c|c|c|c}
$A$ & $B$ & Indecomposable & Reference \\
\hline$a \mathrm{Id}_{4}$ & $-a$ & none & Lemma 2.7 \\
\hline$a \mathrm{Id}_{3}$ & $-a \mathrm{Id}_{2}$ & $\mathcal{D}_{4, a}^{5}$ & Lemma 2.8 \\
\hline $\mathrm{J}_{4}(a)$ & $-a$ & $\mathcal{E}_{1, a}^{5}, \mathcal{E}_{2, a}^{5}$ or $\mathcal{E}_{3, a, b}^{5}$ & Lemma 2.10 \\
\hline $\mathrm{J}_{3}(a)$ & $\mathrm{J}_{2}(-a)$ & $\begin{array}{c}\mathcal{F}_{j, a}^{5}, j \in \mathbb{I}_{3}, \mathcal{F}_{4, a}^{5, i}, \mathcal{F}_{j, a, b, c}^{5} \\
j=5,7, \text { or } \mathcal{F}_{j, a, b, c}^{5, i}, j=6,8\end{array}$ & Lemma 2.11 \\
\hline $\mathrm{J}_{3}(a)$ & $-a \mathrm{Id}_{2}$ & $\mathcal{G}_{a}^{5}$ & Lemma 2.12 \\
\hline$\left(\begin{array}{c}\mathrm{J}_{3}(a) \\
(a)\end{array}\right)$ & $-a$ & $\mathcal{H}_{1, a}^{5}, \mathcal{H}_{2, a}^{5}$ or $\mathcal{H}_{3, a, b}^{5}$ & Lemma 2.13 \\
\hline$\left(\begin{array}{c}\mathrm{J}_{2}(a) \\
\mathrm{J}_{2}(a)\end{array}\right)$ & $-a$ & $\mathcal{I}_{a}^{5}$ & Lemma 2.14 \\
\hline
\end{tabular}

2.4. Tensor products of some indecomposable modules. Here we start the study of the tensor category $\operatorname{rep} L$. Let $\mathfrak{F}$ be a family of isomorphism classes of indecomposable modules. Let $\operatorname{rep}_{\mathfrak{F}} L$ be the full subcategory of rep $L$ whose objects are direct sums of representatives of $\mathfrak{F}$, or in other words whose indecomposable components belong to $\mathfrak{F}$.

Proposition 2.19. Let $\mathfrak{F}$ be the family of classes of the modules $\mathbb{k}_{a}, \mathcal{U}_{a, b}$, $\mathcal{W}_{a}, a, b \in \mathbb{R}^{\times}$. Then $\operatorname{rep}_{\mathfrak{F}} L$ is a monoidal subcategory of $\operatorname{rep} L$.

Proof. Let $a, b, c, d \in \mathbb{k}^{\times}$. First we claim the results summarized in the following table; the proofs are either straightforward or else appear below. 


\begin{tabular}{c|ccc} 
& $\mathbb{k}_{b}$ & $\mathcal{U}_{1, d}$ & $\mathcal{W}_{1}$ \\
\hline$\left(\_\right)^{*}$ & $\mathbb{k}_{b^{-1}}$ & $\mathcal{U}_{1,-d}$ & $\mathcal{W}_{-1}$ \\
\hline $\mathbb{k}_{a} \otimes_{-}$ & $\mathbb{k}_{a b}$ & $\mathcal{U}_{a, a^{2} d}$ & $\mathcal{W}_{a}$ \\
\hline $\mathcal{U}_{1, c} \otimes_{-}$ & $\mathcal{U}_{b, c}$ & $\mathcal{U}_{1, c+d} \oplus \mathcal{U}_{1, c+d}$, if $c \neq-d$ & $\mathcal{U}_{1, c} \oplus \mathcal{U}_{1, c}$ \\
& \multicolumn{3}{|c}{$\mathcal{W}_{1} \oplus \mathcal{W}_{-1}$, if $c=-d$} \\
\hline $\mathcal{W}_{1} \otimes_{-}$ & $\mathcal{W}_{b}$ & $\mathcal{U}_{-1, d} \oplus \mathcal{U}_{-1, d}$ & $\mathcal{W}_{1} \oplus \mathcal{W}_{-1}$
\end{tabular}

Next, we claim that (2.6) implies the Proposition. For instance,

$$
\begin{aligned}
& \mathcal{U}_{a, c} \otimes \mathcal{U}_{b, d} \simeq \mathcal{U}_{1, c} \otimes \mathbb{k}_{a} \otimes \mathcal{U}_{1, d} \otimes \mathbb{k}_{b} \simeq \mathcal{U}_{1, c} \otimes \mathcal{U}_{a, a^{2} d} \otimes \mathbb{k}_{b} \\
& \simeq \mathcal{U}_{1, c} \otimes \mathcal{U}_{1, a^{2} d} \otimes \mathbb{k}_{a b} \simeq \begin{cases}\mathcal{U}_{a b, c+a^{2} d} \oplus \mathcal{U}_{a b, c+a^{2} d}, & c \neq a^{2} d, \\
\mathcal{W}_{-a b} \oplus \mathcal{W}_{a b}, & c=a^{2} d\end{cases}
\end{aligned}
$$

We notice that $\operatorname{rep}_{\mathfrak{F}} L$ has interesting monoidal subcategories by considering some subsets of parameters, e. g. $a, b, c, d$ in a subring of $\mathbb{k}$, with $a, b$ units. Also notice that $\operatorname{rep}_{\mathfrak{F}} L=\oplus_{\lambda \in \mathbb{k}} \times \operatorname{rep}_{\mathfrak{F}}^{\lambda} L$, where $\operatorname{rep}_{\mathfrak{F}}^{\lambda} L:=\operatorname{rep}_{\mathfrak{F}} L \cap \operatorname{rep}^{\lambda} L$. In particular, $\operatorname{rep}_{\mathfrak{F}}^{1} L$ appears to be interesting.

Remark 2.20. The monoidal category $\operatorname{rep}_{\mathfrak{F}} L$ can be interpreted as follows.

(i) Let $\mathrm{A}$ be a Hopf algebra with the Chevalley property, i.e. the tensor product of any two simple A-modules is semisimple. Then the full subcategory rep $_{\mathrm{ssi}} \mathrm{A}$ of rep A consisting of semisimple modules is monoidal. Clearly, is the category of comodules over the Hopf subalgebra of the Sweedler dual of A generated by the matrix coefficients of simple modules. But it is not a Serre subcategory of rep A, in general. For instance, if $\Gamma$ is an abelian group and $A=\mathbb{k} \Gamma$, then $\operatorname{rep}_{\mathrm{ssi}} A$ is the category of $\mathbb{k} \widehat{\Gamma}$-comodules.

(ii) Now assume that $A$ is a Hopf subalgebra of a Hopf algebra $B$ and let $\mathfrak{C}$ be a monoidal subcategory of rep $A$. Then the full subcategory $\operatorname{rep}_{\mathfrak{C}} A$ of rep $\mathrm{B}$ consisting of $\mathrm{B}$-modules that when restricted to $\mathrm{A}$ belong to $\mathfrak{C}$ is monoidal.

Then $\operatorname{rep}_{\mathfrak{F}} L$ is a monoidal subcategory of $\operatorname{rep}_{\mathfrak{C}} L$, where $\mathfrak{C}$ is $\operatorname{rep}_{\mathrm{ssi}} \mathbb{k} G$.

2.4.1. Two simple modules, $\operatorname{dim} 2$. Let $c, d \in \mathbb{k}^{\times}$and $V=\mathcal{U}_{1, c} \otimes \mathcal{U}_{1, d}$. Let $v_{1}, v_{2}$ be a basis of $\mathcal{U}_{1, c}, w_{1}, w_{2}$ a basis of $\mathcal{U}_{1, d}$, both realizing (2.2). In the basis $u_{1}=v_{1} \otimes w_{1}, u_{2}=v_{2} \otimes w_{2}, u_{3}=v_{1} \otimes w_{2}, u_{4}=v_{2} \otimes w_{1}$, the action is

$$
\begin{aligned}
& g u_{1}=u_{1}, \quad y u_{1}=u_{3}+u_{4}, \quad g u_{3}=-u_{3}, \quad y u_{3}=d u_{1}+u_{2}, \\
& g u_{2}=u_{2}, \quad y u_{2}=c u_{3}-d u_{4}, \quad g u_{4}=-u_{4}, \quad y u_{4}=c u_{1}-u_{2} .
\end{aligned}
$$

We claim that

$$
\begin{aligned}
c+d \neq 0 & \Longrightarrow \mathcal{U}_{1, c} \otimes \mathcal{U}_{1, d} \simeq \mathcal{U}_{1, c+d} \oplus \mathcal{U}_{1, c+d} \\
c+d=0 & \Longrightarrow \mathcal{U}_{1, c} \otimes \mathcal{U}_{1, d} \simeq \mathcal{W}_{-1} \oplus \mathcal{W}_{1} .
\end{aligned}
$$


Proof. $c+d \neq 0$ : Here $u_{1}, u_{3}+u_{4}, u_{2}, c u_{3}-d u_{4}$ is a basis of $V$. By (2.7), $\left\langle u_{1}, u_{3}+u_{4}\right\rangle \leq V$ and $\left\langle u_{2}, c u_{3}-d u_{4}\right\rangle \leq V$, both isomorphic to $\mathcal{U}_{1, c+d}$.

$c+d=0$ : Now $u_{3}+u_{4}, u_{1}, d u_{1}+u_{2}, u_{3}$ is a basis of $V$. By (2.7), $\mathcal{W}_{-1} \simeq\left\langle u_{3}+u_{4}, u_{1}\right\rangle \leq V$ and $\mathcal{W}_{1} \simeq\left\langle d u_{1}+u_{2}, u_{3}\right\rangle \leq V$.

2.4.2. Simple and indecomposable, $\operatorname{dim} 2$. Let $c, d \in \mathbb{k}^{\times}$and $V=\mathcal{U}_{1, c} \otimes \mathcal{W}_{1}$. Let $v_{1}, v_{2}$ be a basis of $\mathcal{U}_{1, c}, w_{1}, w_{2}$ a basis of $\mathcal{W}_{1}$. In the basis $u_{1}=v_{1} \otimes w_{1}$, $u_{2}=v_{2} \otimes w_{2}, u_{3}=v_{1} \otimes w_{2}, u_{4}=v_{2} \otimes w_{1}$ of $V$ the action is

$$
\begin{aligned}
& g u_{1}=u_{1}, \quad y u_{1}=u_{4}, \quad g u_{3}=-u_{3}, \quad y u_{3}=u_{1}+u_{2}, \\
& g u_{2}=u_{2}, \quad y u_{2}=c u_{3}-u_{4}, \quad g u_{4}=-u_{4}, \quad y u_{4}=c u_{1} \text {. }
\end{aligned}
$$

We claim that

$$
\mathcal{U}_{1, c} \otimes \mathcal{W}_{1} \simeq \mathcal{U}_{1, c} \oplus \mathcal{U}_{1, c}, \quad \mathcal{W}_{1} \otimes \mathcal{U}_{1, d} \simeq \mathcal{U}_{-1, d} \oplus \mathcal{U}_{-1, d} .
$$

Proof. By (2.9), $\left\langle u_{1}, u_{4}\right\rangle \leq V$ and $\left\langle u_{2}, c u_{3}-u_{4}\right\rangle \leq V$, both $\simeq \mathcal{U}_{1, c}$, hence the first isomorphism. Next compute $\mathbb{k}_{-1} \otimes\left(\mathcal{U}_{1, c} \otimes \mathcal{W}_{1}\right)^{*} \simeq \mathbb{k}_{-1} \otimes\left(\mathcal{U}_{1, c} \oplus \mathcal{U}_{1, c}\right)^{*}$ :

$$
\begin{aligned}
\mathbb{k}_{-1} \otimes\left(\mathcal{W}_{1}^{*} \otimes \mathcal{U}_{1, c}^{*}\right) & \simeq \mathbb{k}_{-1} \otimes\left(\mathcal{W}_{-1} \otimes \mathcal{U}_{1,-c}\right) \simeq \mathbb{k}_{-1} \otimes\left(\mathcal{U}_{1,-c} \oplus \mathcal{U}_{1,-c}\right) \\
& \Longrightarrow \mathcal{W}_{1} \otimes \mathcal{U}_{1,-c} \simeq \mathcal{U}_{-1,-c} \oplus \mathcal{U}_{-1,-c} .
\end{aligned}
$$

2.4.3. Two indecomposable modules of dimension 2. Let $v_{1}, v_{2}$ and $w_{1}, w_{2}$ be basis of two copies of $\mathcal{W}_{1}$. In the basis $u_{1}=v_{1} \otimes w_{1}, u_{2}=v_{2} \otimes w_{2}$, $u_{3}=v_{1} \otimes w_{2}, u_{4}=v_{2} \otimes w_{1}$ of $V=\mathcal{W}_{1} \otimes \mathcal{W}_{1}$ the action is

$$
\begin{aligned}
& g u_{1}=u_{1}, \quad y u_{1}=0, \quad g u_{3}=-u_{3}, \quad y u_{3}=u_{1}, \\
& g u_{2}=u_{2}, \quad y u_{2}=u_{3}-u_{4}, \quad g u_{4}=-u_{4}, \quad y u_{4}=u_{1} .
\end{aligned}
$$

We claim that

$$
\mathcal{W}_{1} \otimes \mathcal{W}_{1} \simeq \mathcal{W}_{1} \oplus \mathcal{W}_{-1}
$$

Proof. By (2.11), $\mathcal{W}_{1} \simeq\left\langle u_{1}, u_{3}\right\rangle \leq V$ and $\mathcal{W}_{-1} \simeq\left\langle u_{3}-u_{4}, u_{2}\right\rangle \leq V$.

\section{The Bosonization of the Jordan Plane}

Let $\mathcal{A}=\mathbb{k}\left\langle\mathbf{y}_{1}, \mathbf{y}_{2}\right\rangle$ modulo the ideal generated by the quadratic relation

$$
\mathbf{y}_{2} \mathbf{y}_{1}-\mathbf{y}_{1} \mathbf{y}_{2}+\frac{1}{2} \mathbf{y}_{1}^{2} \text {. }
$$

This is the well-known Jordan plane. Let $\mathbf{G}$ be an infinite cyclic group denoted multiplicatively with a fixed generator g. Let $\mathcal{V}=\mathbb{k} \mathbf{y}_{1} \oplus \mathbb{k} \mathbf{y}_{2} \in$

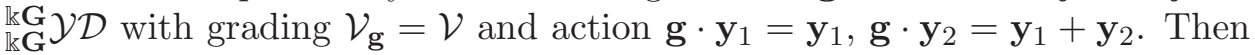
$\mathcal{A} \simeq \mathscr{B}(\mathcal{V})$, cf. AAH2, Prop. 3.4]. Let $H:=\mathcal{A} \# \mathbb{k} \mathbf{G}$ the bosonization of $\mathcal{A}$ by $\mathbb{k} \mathbf{G}$; i. e. $H=\mathbb{k}\left\langle\mathbf{y}_{1}, \mathbf{y}_{2}, \mathbf{g}^{ \pm 1}\right\rangle$ modulo the ideal generated by (3.1), $\mathbf{g}^{ \pm} \mathbf{g}^{\mp}-1$

$$
\begin{aligned}
& \mathbf{g y}_{1} \mathbf{g}^{-1}-\mathbf{y}_{1}, \\
& \mathbf{g y}_{2} \mathbf{g}^{-1}-\left(\mathbf{y}_{1}+\mathbf{y}_{2}\right) .
\end{aligned}
$$


This is a Hopf algebra with the comultiplication determined by

$$
\Delta\left(\mathbf{g}^{ \pm 1}\right)=\mathbf{g}^{ \pm 1} \otimes \mathbf{g}^{ \pm 1}, \quad \Delta\left(\mathbf{y}_{i}\right)=\mathbf{y}_{i} \otimes 1+\mathbf{g} \otimes \mathbf{y}_{i}, \quad i=1,2 .
$$

The set $\left\{\mathbf{y}_{1}^{a} \mathbf{y}_{2}^{b} \mathbf{g}^{c}: a, b \in \mathbb{N}_{0}, c \in \mathbb{Z}\right\}$ is a basis of $H$, whose GKdim is 3 .

3.1. The Hopf algebra $\bar{H}$. From the defining relations, we see that the left ideal $H \mathbf{y}_{1}$ is a two-sided, as well as a Hopf ideal. Then $\bar{H}:=H / H \mathbf{y}_{1}$ is the commutative Hopf algebra $\mathbb{k}\left\langle\overline{\mathbf{g}}^{ \pm 1}, \overline{\mathbf{y}}_{2}\right\rangle$, that is

$$
\bar{H} \simeq \mathcal{O}(\mathbb{B}), \quad \mathbb{B}=\left\{\left(\begin{array}{cc}
a & b \\
0 & a^{-1}
\end{array}\right):(a, b) \in \mathbb{k}^{\times} \times \mathbb{k}\right\} \leq S L_{2}(\mathbb{k}),
$$

where $\mathcal{O}$ stands for the algebra of regular functions. Therefore, the tensor category rep $\bar{H}$ reflects the group structure of $\mathbb{B}$. The $\bar{H}$-modules, are described as follows. Let $A \in \mathrm{GL}_{n}(\mathbb{k})$ and $B \in \operatorname{End}\left(\mathbb{k}^{n}\right)$ such that $A B=B A$. We denote by $\mathbb{k}_{A, B}^{n} \in \operatorname{rep} \bar{H}$ the vector space $\mathbb{k}^{n}$ with action given by $\overline{\mathbf{g}} \mapsto A$ and $\overline{\mathbf{y}}_{2} \mapsto B$. Every $V \in \operatorname{rep} \bar{H}$ is isomorphic to some $\mathbb{k}_{A, B}^{n}$; also, $\mathbb{k}_{A, B}^{n} \simeq \mathbb{k}_{A^{\prime}, B^{\prime}}^{n}$ iff $A, B$ and $A^{\prime}, B^{\prime}$ are simultaneously conjugated. For $n=1$, let $\mathbb{k}_{\gamma}=\mathbb{k}_{a, b}$, where $\gamma=\left(\begin{array}{cc}a & b \\ 0 & a^{-1}\end{array}\right) \in \mathbb{B}$; this says that the simple $\bar{H}$-modules are classified by the points of $\mathbb{B}$. Given $a \in \mathbb{k}^{\times}, b, c \in \mathbb{k}$, we have $\bar{H}$-modules of dimension 2 given by

$$
\mathcal{J}_{a, b}: \mathbf{g} \mapsto\left(\begin{array}{cc}
a & 0 \\
0 & a
\end{array}\right), \quad \overline{\mathbf{y}}_{2} \mapsto\left(\begin{array}{cc}
b & 1 \\
0 & b
\end{array}\right), \quad \mathcal{K}_{a, b, c}: \overline{\mathbf{g}} \mapsto\left(\begin{array}{cc}
a & 1 \\
0 & a
\end{array}\right), \quad \overline{\mathbf{y}}_{2} \mapsto\left(\begin{array}{cc}
b & c \\
0 & b
\end{array}\right) .
$$

Clearly these are pairwise non-isomorphic indecomposable $\bar{H}$-modules. We leave to the reader the (elementary) proof of the following result.

Lemma 3.1. (i) If $V \in \operatorname{rep} \bar{H}$ is indecomposable of dimension 2, then either $V \simeq \mathcal{J}_{a, b}$, or $V \simeq \mathcal{K}_{a, b, c}$ for unique $a, b, c$.

(ii) $\operatorname{dim} \operatorname{Ext} \frac{1}{H}\left(\mathbb{k}_{\gamma}, \mathbb{k}_{\eta}\right)=\delta_{\gamma, \eta}$.

(iii) If $a \in \mathbb{k}^{\times}$and $b, c \in \mathbb{k}$, then

$$
\begin{array}{rlrl}
\mathbb{k}_{a, b-a} \otimes \mathcal{J}_{1,1} & \simeq \mathcal{J}_{a, b} \simeq \mathcal{J}_{1,1} \otimes \mathbb{k}_{a, b-1}, & \mathcal{J}_{a, b}^{*} & \simeq \mathcal{J}_{a^{-1},-b a^{-1}} \\
\mathbb{k}_{a, b-a} \otimes \mathcal{K}_{1,1, c} & \simeq \mathcal{K}_{a, b, c} \simeq \mathcal{K}_{1,1, a c-(b-1)} \otimes \mathbb{k}_{a, b-1}, & \mathcal{K}_{a, b, c}^{*} \simeq \mathcal{K}_{a^{-1},-b a^{-1}, c a-b} .
\end{array}
$$

3.2. The category rep $H$. The projection $H \rightarrow \bar{H}$ induces a functor $\operatorname{rep} \bar{H} \rightarrow \operatorname{rep} H$. We carry over the notation along this functor. Conversely, let $V \in \operatorname{rep} H$ and $V_{0}:=\operatorname{ker} \mathbf{y}_{1}$, giving a functor $\operatorname{rep} H \rightarrow \operatorname{rep} \bar{H}$. If $V=V_{0}$ has dimension $n$, then $V \in \operatorname{rep} \bar{H}$, hence $V \simeq \mathbb{k}_{A, B}^{n}$ for some $A, B$.

Let $V \in \operatorname{rep} H$. Then $V_{0} \leq V$ since $\mathbf{y}_{1}\left(\mathbf{y}_{2} V_{0}\right)=0$ by (3.1), and $\mathbf{y}_{1}\left(\mathbf{g} V_{0}\right)=$ 0 by (3.2).

Lemma 3.2. [I, Lemma 2.1] If $U \in \operatorname{rep} \mathcal{A}$, then $\mathbf{y}_{1}$ acts nilpotently on $U$; in particular $V_{0} \neq 0$.

The Lemma applies to $V \in \operatorname{rep} H$ via the evident restriction functor. 
Proposition 3.3. If $V \in \operatorname{rep} H$ is irreducible, then $V \simeq \mathbb{k}_{\gamma}$, for a unique $\gamma \in \mathbb{B}$.

We next classify the indecomposable $H$-modules of dimension 2 .

Proposition 3.4. Let $V \in \operatorname{rep} H, \operatorname{dim} V=2$. Then $V=V_{0}$. Therefore, if $V$ is indecomposable, then either $V \simeq \mathcal{J}_{a, b}$, or $V \simeq \mathcal{K}_{a, b, c}$ for unique $a, b, c$. Also

$$
\operatorname{dim} \operatorname{Ext}_{H}^{1}\left(\mathbb{k}_{\gamma}, \mathbb{k}_{\eta}\right)=\delta_{\gamma, \eta} .
$$

Proof. If $V \neq V_{0}$ then there exists a basis $\Omega$ of $V$ such that

$$
\left[\mathbf{y}_{1}\right]_{\Omega}=\left(\begin{array}{ll}
0 & 1 \\
0 & 0
\end{array}\right), \quad[\mathbf{g}]_{\Omega}=\left(\begin{array}{ll}
a & b \\
c & d
\end{array}\right), \quad\left[\mathbf{y}_{2}\right]_{\Omega}=\left(\begin{array}{ll}
e & f \\
h & i
\end{array}\right) .
$$

By (3.2), $c=0$ and $a=d$. From $\mathbf{y}_{1} \mathbf{y}_{2}=\mathbf{y}_{2} \mathbf{y}_{1}$ we see that $h=0$ and $e=i$. By (3.3), we conclude that $a f+b e=a(f+1)+b e$. Hence, $a=0$ and $\mathbf{g}$ is not invertible, a contradiction. The last claims follow from Lemma 3.1 .

\section{The Bosonization of the SUper Jordan Plane}

Let $x_{21}=x_{1} x_{2}+x_{2} x_{1}$ in the free associative algebra in generators $x_{1}$ and $x_{2}$. Let $\mathcal{B}$ be the algebra generated by $x_{1}$ and $x_{2}$ with defining relations

$$
\begin{aligned}
& x_{1}^{2}, \\
& x_{2} x_{21}-x_{21} x_{2}-x_{1} x_{21} .
\end{aligned}
$$

The algebra $\mathcal{B}$, introduced in [AAH2], is called the super Jordan plane. Let $\mathcal{V}^{\prime}=\mathbb{k} x_{1} \oplus \mathbb{k} x_{2} \in \mathbb{k}_{\mathbb{k} G} \mathcal{Y} \mathcal{D}$ with grading $\mathcal{V}_{g}^{\prime}=\mathcal{V}^{\prime}$ and action $g \cdot x_{1}=-x_{1}$, $g \cdot x_{2}=-x_{1}+x_{2}$. Then $\mathcal{B} \simeq \mathscr{B}\left(\mathcal{V}^{\prime}\right)$, cf. AAH2, Prop. 3.5]. Let $K:=\mathcal{B} \# \mathbb{k} G$ the bosonization of $\mathcal{B}$ by $\mathbb{k} G$; i. e. $K=\mathbb{k}\left\langle x_{1}, x_{2}, g^{ \pm 1}\right\rangle$ modulo the ideal generated by (4.1), (4.2), $g^{ \pm} g^{\mp}-1$,

$$
\begin{aligned}
& g x_{1} g^{-1}+x_{1}, \\
& g x_{2} g^{-1}-x_{1}+x_{2} .
\end{aligned}
$$

This is a Hopf algebra with the comultiplication determined by

$$
\Delta\left(g^{ \pm 1}\right)=g^{ \pm 1} \otimes g^{ \pm 1}, \quad \Delta\left(x_{i}\right)=x_{i} \otimes 1+g \otimes x_{i}, \quad i=1,2 .
$$

The set $\left\{x_{1}^{a} x_{21}^{b} x_{2}^{c} g^{d}: a \in \mathbb{I}_{0,1}, b, c \in \mathbb{N}_{0}, d \in \mathbb{Z}\right\}$ is a basis of $K$, whose GKdim is 3 . The following identities are valid in $K$ :

$$
\begin{aligned}
x_{21} x_{1} & =x_{1} x_{21}, \\
x_{2}^{2} x_{1} & =x_{1} x_{2}^{2}+x_{1} x_{2} x_{1}, \\
x_{21} x_{2}^{2} & =\left(x_{2}^{2}-x_{21}\right) x_{21}, \\
g x_{21} & =x_{21} g, \\
g x_{2}^{2} & =\left(x_{2}^{2}-x_{21}\right) g .
\end{aligned}
$$


Lemma 4.1. (i) There is an injective algebra map $\varphi: H \rightarrow K$ given by

$$
\varphi\left(\mathbf{y}_{1}\right)=x_{21}, \quad \varphi\left(\mathbf{y}_{2}\right)=-\frac{1}{2} x_{2}^{2}, \quad \varphi(\mathbf{g})=g^{2} .
$$

(ii) $K x_{1} K=K x_{1}+K x_{21}$ is a Hopf ideal and $K / K x_{1} K \simeq L$.

Proof. (i) It is not difficult to check that $\varphi$ is well-defined; indeed (3.1) follows from (4.7), (3.2) from (4.8) and (3.3) from (4.9). The injectivity is verified using the PBW-bases.

(ii) Let $r=x_{2}-x_{1}$. We prove by induction on $n$ that

$$
x_{1} x_{2}^{n}=(-1)^{n} x_{2}^{n} x_{1}+\left((-1)^{n-1} x_{2}^{n-1}+\cdots+x_{2}^{2} r^{n-3}-x_{1} r^{n-2}\right) x_{21} .
$$

For $n=1$, (4.11) is trivial. If $n>1$ and (4.11) holds for $n-1$, then $x_{1} x_{2}^{n}=$

$$
\begin{aligned}
& =\left\{(-1)^{n-1} x_{2}^{n-1} x_{1}+\left((-1)^{n-2} x_{2}^{n-2}+\cdots+x_{2}^{2} r^{n-4}-x_{1} r^{n-3}\right) x_{21}\right\} x_{2} \\
& =(-1)^{n-1} x_{2}^{n-1} x_{1} x_{2}+\left((-1)^{n-2} x_{2}^{n-2}+\cdots+x_{2}^{2} r^{n-4}-x_{1} r^{n-3}\right) x_{21} x_{2} \\
& =(-1)^{n-1} x_{2}^{n-1}\left(x_{21}-x_{2} x_{1}\right)+\left((-1)^{n-2} x_{2}^{n-2}+\cdots+x_{2}^{2} r^{n-4}-x_{1} r^{n-3}\right) r x_{21} \\
& =(-1)^{n} x_{2}^{n} x_{1}+\left((-1)^{n-1} x_{2}^{n-1}+\cdots+x_{2}^{2} r^{n-3}-x_{1} r^{n-2}\right) x_{21} .
\end{aligned}
$$

Hence $x_{1} x_{2}^{n} \in K x_{1}+K x_{21}$, for all $n \in \mathbb{N}$, and $x_{1} K \subset K x_{1}+K_{21}$. The isomorphism is verified using the PBW-bases.

4.1. Relations between $\operatorname{rep} K, \operatorname{rep} L$ and $\operatorname{rep} H$. Let $V \in \operatorname{rep} K$. If $x_{1}=$ 0 in $V$, then also $x_{21}=0$; by Lemma 4.1, we conclude that $V \in \operatorname{rep} L$, a category discussed in 92 . Hence we may assume that $x_{1} \neq 0$.

More generally, since $x_{1}^{2}=0$, we may think on it as a differential on $V$ and consider its homology. Namely, define

$$
\mathscr{K}(V)=\operatorname{ker} x_{1} \text { on } V, \quad \mathscr{I}(V)=x_{1}(V), \quad \mathscr{H}(V)=\operatorname{Im}(V) / \mathscr{K}(V) .
$$

Proposition 4.2. (i) $\mathscr{K}, \mathscr{I}, \mathscr{H}$ are $\mathbb{k}$-linear functors rep $K \rightarrow \operatorname{rep} H$.

(ii) $\mathscr{K}$ is left exact.

(iii) $\mathscr{I}$ is right exact.

Proof. (i) The claims for $\mathscr{K}, \mathscr{I}$ are consequences of (4.5) and (4.6), and imply in turn that of $\mathscr{H}$. (ii) and (iii) are standard.

4.2. Simple modules. We show that the classification of the simple objects in rep $K$ reduces to those in rep $L$ given in Proposition 2.1.

Theorem 4.3. Let $V \in \operatorname{rep} K$ irreducible. Then $V \in \operatorname{rep} L$, in particular $\operatorname{dim} V=1$ or $\operatorname{dim} V=2$.

Proof. First we claim that $W:=\operatorname{ker} x_{21}$ is a submodule of $V$. By (4.6) and (4.8), $W$ is stable by $x_{1}$ and $g$. Let $u \in W$ so that $x_{1} x_{2} u=-x_{2} x_{1} u$. Then

$$
x_{21} x_{2} u=x_{1} x_{2}^{2} u+x_{2} x_{1} x_{2} u=x_{1} x_{2}^{2} u-x_{2}^{2} x_{1} u \stackrel{\text { (4.6) }}{=}-x_{1} x_{2} x_{1} u=x_{1}^{2} x_{2} u=0,
$$

hence $x_{2} u \in W$. By Lemmas 3.2 and 4.1 (i), $W \neq 0$ hence $W=V$, i. e. $x_{1} x_{2}=-x_{2} x_{1}$ in $V$. Thus $0 \neq \operatorname{ker} x_{1}$ is $x_{2}$-stable and by (4.3), ker $x_{1} \leq V$; consequently ker $x_{1}=V$. 
4.3. Indecomposable modules. For convenience, we set

$$
s=x_{21}, \quad t=x_{2}^{2} .
$$

Let $V \in \operatorname{rep} K$ such that $x_{1} \neq 0$ on $V$. By Lemmas 3.2 and 4.1, $s$ is nilpotent. The aim of this Subsection is to establish the following result.

Proposition 4.4. Let $V \in \operatorname{rep} K$ such that $x_{1} \neq 0$.

(i) $V_{t}^{(a)} \leq V, a \in \mathbb{k}$; hence $V=\oplus_{a \in \mathbb{k} \times} V_{t}^{(a)}$ is a decomposition in rep $K$.

(ii) If $V$ is indecomposable, then $V=V_{t}^{(a)}$, for a unique eigenvalue a of t. Hence spec $x_{2} \subseteq\{ \pm \nu\}$, where $\nu^{2}=a$.

(iii) Let $V[\lambda]:=V_{g}^{(b)} \oplus V_{g}^{(-b)}, \lambda=b^{2} \in \mathbb{k}^{\times}$. Then $V[\lambda] \leq V$, hence $V=\oplus_{\lambda \in \mathbb{k} \times} \times[\lambda]$ is a decomposition in $\operatorname{rep} K$.

(iv) If $V$ is indecomposable, then $V=V[\lambda]$, for a unique $\lambda=b^{2} \in \mathbb{k}^{\times}$and $\operatorname{spec} g=\{ \pm b\}$.

We start by relations in $\mathcal{B}$ that might be useful for other problems. Set

$$
\zeta_{n, j}=\frac{n !}{(n-j) !}, \quad j, n \in \mathbb{N}_{0}, j \leq n .
$$

Lemma 4.5. Let $a, b \in \mathbb{k}$ and set $w=s-a, z=t-a \in \mathcal{B}$. Then for every $n \in \mathbb{N}$, we have

$$
\begin{aligned}
z^{n} x_{1} & =x_{1} \sum_{j \in \mathbb{I}_{0, n}} \zeta_{n, j} s^{j} z^{n-j} \\
z^{n} g & =g(z+s)^{n} \\
(z+s)^{n} & =\sum_{j \in \mathbb{I}_{0, n}} \zeta_{n, j} s^{j} z^{n-j}, \\
w^{n} x_{2} & =x_{2} w^{n}-n x_{1} s w^{n-1} . \\
(g-b)^{n} x_{1} & =(-1)^{n} x_{1}(g+b)^{n}, \\
(g-b)^{n} x_{2} & =(-1)^{n-1}\left(n x_{1} g-x_{2}(g+b)\right)(g+b)^{n-1} .
\end{aligned}
$$

Proof. (4.13) is [ABDF, Lemma 2.9]. (4.14): $x_{2} g=-g\left(x_{1}+x_{2}\right)$ by (4.3) and (4.4). Hence $t g=x_{2}^{2} g=g\left(x_{1}+x_{2}\right)^{2}=g\left(x_{21}+x_{2}^{2}\right)=g(s+t)$. Thus $z g=g(z+s)$ and $z^{n} g=g(z+s)^{n}$.

(4.15): We proceed by induction on $n$; the case $n=1$ is trivial. Let $n>1$. By (4.7), $z s=s z+s^{2}$ whence $z s^{n}=s^{n} z+n s^{n+1}$. Then

$$
\begin{aligned}
(z+s)^{n} & =(z+s) \sum_{j=0}^{n-1} \zeta_{n-1, j} s^{j} z^{n-1-j}=\sum_{j=0}^{n-1} \zeta_{n-1, j}\left(z s^{j} z^{n-1-j}+s^{j+1} z^{n-1-j}\right) \\
& =\sum_{j=0}^{n-1} \zeta_{n-1, j}\left(s^{j} z^{n-j}+j s^{j+1} z^{n-1-j}+s^{j+1} z^{n-1-j}\right) \\
& =z^{n}+\sum_{k=1}^{n-1}\left(\zeta_{n-1, k}+k \zeta_{n-1, k-1}\right) s^{k} z^{n-k}+n ! s^{n}=\sum_{k=0}^{n} \zeta_{n, k} s^{k} z^{n-k} .
\end{aligned}
$$


(4.16): also by induction on $n$. For $n=1$, we have

$$
\begin{aligned}
w x_{2} & =x_{1} x_{2}^{2}+x_{2} x_{1} x_{2}-a x_{2} \stackrel{\text { 4.6) }}{=} x_{2}^{2} x_{1}-x_{1} x_{2} x_{1}+x_{2} x_{1} x_{2}-a x_{2} \\
& =x_{2}(s-a \mathrm{id})-x_{1} s=x_{2} w-x_{1} s .
\end{aligned}
$$

Let $n>1$ and suppose that the identity is true for $n-1$. Then

$$
\begin{aligned}
w^{n} x_{2} & =w\left(x_{2} w^{n-1}-(n-1) x_{1} s w^{n-2}\right) \\
& =\left(x_{2} w-x_{1} s\right) w^{n-1}-(n-1) x_{1} s w^{n-1}=x_{2} w^{n}-n x_{1} s w^{n-1} .
\end{aligned}
$$

(4.17) follows at once from (4.3). (4.18): For $n=1$, we have

$$
(g-b) x_{2}=g x_{2}-b x_{2} \stackrel{\text { (4.4) }}{=}\left(x_{1}-x_{2}\right) g-b x_{2}=x_{1} g-x_{2}(g+b) .
$$

For $n>1$ we compute

$$
\begin{aligned}
(g-b)^{n} x_{2} & =(-1)^{n-2}(g-b)\left\{(n-1) x_{1} g-x_{2}(g+b)\right\}(g+b)^{n-2} \\
& =(-1)^{n-2}\left\{-(n-1) x_{1} g-x_{1} g+x_{2}(g+b)\right\}(g+b)^{n-1} \\
& =(-1)^{n-1}\left(n x_{1} g-x_{2}(g+b)\right)(g+b)^{n-1} .
\end{aligned}
$$

Proof of Proposition 4.4. (i). Clearly, $V_{t}^{(a)}$ is stable by $x_{2}$. By (4.13), (4.14) and (4.15), with $n=2 \operatorname{dim} V$, it is stable by $x_{1}$ and $g$. (ii) follows from (i).

(iii)] By (4.17), respectively (4.18), $x_{1} \cdot V_{g}^{( \pm b)} \subset V_{g}^{(\mp b)}$ and $x_{2} \cdot V_{g}^{( \pm b)} \subset$ $V_{g}^{(\mp b)}$. Hence $V[\lambda] \leq V$, implying the first claim in (iv), By (4.17), since $x_{1} \neq 0$, there exists $b \in \operatorname{spec} g$ such that $V_{g}^{(-b)} \neq 0$, thus $-b \in \operatorname{spec} g$.

Let $V \in \operatorname{rep} K$ and $\lambda=a^{2} \in \mathbb{k}^{\times}$such that $V=V[\lambda]$. Let $\mathfrak{B}_{ \pm}$be a basis of $V_{g}^{( \pm a)}$ and $\mathfrak{B}:=\mathfrak{B}_{+} \cup \mathfrak{B}_{-}$; let $\ell=\operatorname{dim} V_{g}^{(a)}, \wp=\operatorname{dim} V-\ell$. Then

$$
[g]_{\mathfrak{B}}=\left(\begin{array}{cc}
A & 0 \\
0 & B
\end{array}\right), \quad\left[x_{1}\right]_{\mathfrak{B}}=\left(\begin{array}{cc}
0 & C \\
D & 0
\end{array}\right), \quad\left[x_{2}\right]_{\mathfrak{B}}=\left(\begin{array}{cc}
0 & E \\
F & 0
\end{array}\right),
$$

where $A \in \mathbf{G L}_{\ell}(\mathbb{k}), B \in \mathbf{G L}_{\wp}(\mathbb{k}), C, E \in \mathrm{M}_{\ell \times \wp}(\mathbb{k})$ and $D, F \in \mathrm{M}_{\wp \times \ell}(\mathbb{k})$. Conversely, $g, x_{1}$ and $x_{2}$ given by (4.19) define a representation of $K$ if and only if

$$
\begin{aligned}
& C D=0, \quad D C=0, \quad C F(C+E)=E F C, \\
& A C=-C B, \quad B D=-D A, \quad(C-E) B=A E, \\
& D E(D+F)=F E D, \quad(D-F) A=B F \text {. }
\end{aligned}
$$

Again we seek to describe conditions that guarantee that $V$ is indecomposable, assuming that $A$ and $B$ are in Jordan form. Actually this will be done in some special cases.

Remark 4.6. Let $V \in \operatorname{rep} K$ indecomposable given by (4.19), $\operatorname{dim} V=n \geq 2$. If $A=a \operatorname{id}_{\ell}$ and $B=-a \operatorname{id}_{\wp}$, then $x_{1}=0$. In fact, from $A E-(C-E) B=0$ follows $a C=0$ hence $C=0$. Similarly, $B F-(D-F) A=0$ implies $D=0$. Particularly, the indecomposable $K$-modules of dimension 2 are just the indecomposable $L$-modules by Proposition 4.4 (iv). 
4.3.1. Representations with $A$ and $B$ Jordan blocks. By the same arguments used in Lemma 2.9 we have the following.

Lemma 4.7. Let $V \in \operatorname{rep} K$ given by (4.19) where $A$ and $B$ are Jordan blocks. Then $V$ is indecomposable if and only if $x_{1} \neq 0$ or $x_{2} \neq 0$.

Assume that $V$ is a $K$-module of dimension $n>3$ such that $x_{1} \neq 0$. Define the representations $\mathcal{L}_{1, a, b}^{n}$ and $\mathcal{L}_{2, a, b}^{n}, a \in \mathbb{k}^{\times}, b \in \mathbb{k}$ by (4.19) where $A=\mathrm{J}_{n-1}(a), B=-a$ and $x_{1}, x_{2}$ acts as follows:

$$
\begin{aligned}
& \mathcal{L}_{1, a, b}^{n}: \quad x_{1} \mapsto\left(\begin{array}{cc}
0 & 0 \\
e_{n-1}^{t} & 0
\end{array}\right), \quad x_{2} \mapsto\left(\begin{array}{cc}
0 & b e_{1} \\
a e_{n-2}^{t} & 0
\end{array}\right) . \\
& \mathcal{L}_{2, a, b}^{n}: \quad x_{1} \mapsto\left(\begin{array}{cc}
0 & e_{1} \\
0 & 0
\end{array}\right), \quad \quad x_{2} \mapsto\left(\begin{array}{cc}
0 & -a e_{2} \\
b e_{n-1}^{t} & 0
\end{array}\right) .
\end{aligned}
$$

Clearly these are pairwise non-isomorphic indecomposable $K$-modules.

Lemma 4.8. Let $n>3$ and $V \in \operatorname{rep} K$ given by (4.19) where $A=\mathrm{J}_{n-1}(a)$ and $B=-a$. Then the following are equivalent:

(i) $V$ is indecomposable,

(ii) $x_{1} \neq 0$ or $x_{2} \neq 0$,

(iii) $V$ is isomorphic to one, and only one, of $\mathcal{L}_{1, a, b}^{n}$ or $\mathcal{L}_{2, a, b}^{n}$, for unique $a \in \mathbb{k}^{\times}, b \in \mathbb{k}$.

Proof. (i) $\Leftrightarrow$ (ii) is Lemma 4.7. We prove that (ii) $\Leftrightarrow$ (iii). Here $C=$ $\sum_{i=1}^{n-1} c_{i} e_{i}, D=\sum_{i=1}^{n-1} d_{i} e_{i}^{t}, E=\sum_{i=1}^{n-1} f_{i} e_{i}$ and $F=\sum_{i=1}^{n-1} h_{i} e_{i}^{t}$ with $c_{i}, d_{i}, f_{i}$, $h_{i} \in \mathbb{k}, i \in \mathbb{I}_{n-1}$. By (4.20), (4.21) and (4.22) we have two possibilities:

$\circ C=0, D=d e_{n-1}^{t}, E=f e_{1}, F=a d e_{n-2}^{t}+h e_{n-1}^{t}$, with $d \in \mathbb{k}^{\times}, f, h \in \mathbb{k}$. $\circ C=c e_{1}, D=0, E=f e_{1}-a c e_{2}, F=h e_{n-1}^{t}$, with $c \in \mathbb{k}^{\times}, f, h \in \mathbb{k}$.

In the first, the basis $e_{1},-h(a d)^{-1} e_{1}+e_{2}, \ldots,-h(a d)^{-1} e_{n-2}+e_{n-1}, d e_{n}$ gives $V \simeq \mathcal{L}_{1, a, d f}^{n}$. In the second, the basis $c e_{1},-f a^{-1} e_{1}+c e_{2}, \ldots,-f a^{-1} e_{n-2}+$ $c e_{n-1}, e_{n}$ gives $V \simeq \mathcal{L}_{2, a, c h}^{n}$.

Now if $n=3$, we define two families of representations of $K$ on the vector space $V$ determined by the following action, for all $a \in \mathbb{k}^{\times}$:

$$
\begin{aligned}
& \mathcal{L}_{1, a}^{3}: \quad g \mapsto\left(\begin{array}{cc}
\mathrm{J}_{2}(a) & 0 \\
0 & -a
\end{array}\right), \quad x_{1} \mapsto\left(\begin{array}{cc}
0 & 0 \\
e_{2}^{t} & 0
\end{array}\right), \quad x_{2} \mapsto\left(\begin{array}{cc}
0 & 0 \\
a e_{1}^{t} & 0
\end{array}\right) \\
& \mathcal{L}_{2, a}^{3}: \quad g \mapsto\left(\begin{array}{cc}
\mathrm{J}_{2}(a) & 0 \\
0 & -a
\end{array}\right), \quad x_{1} \mapsto\left(\begin{array}{cc}
0 & e_{1} \\
0 & 0
\end{array}\right), \quad x_{2} \mapsto\left(\begin{array}{cc}
0 & -a e_{2} \\
0 & 0
\end{array}\right) .
\end{aligned}
$$

Clearly these are pairwise non-isomorphic indecomposable $K$-modules. By a similar argument to the Lemma 4.8 we can prove the following result.

Lemma 4.9. If $V$ is indecomposable, then either $V \simeq \mathcal{L}_{1, a}^{3}$ or $V \simeq \mathcal{L}_{2, a}^{3}$.

Remark 4.10. Clearly, $\left(\mathcal{L}_{2, a}^{3}\right)^{*} \simeq \mathcal{L}_{1, a^{-1}}^{3}, \mathcal{L}_{2,1}^{3} \otimes \mathbb{k}_{a} \simeq \mathcal{L}_{2, a}^{3} \simeq \mathbb{k}_{a} \otimes \mathcal{L}_{2,1}^{3}$ and $\mathcal{L}_{1,1}^{3} \otimes \mathbb{k}_{-a} \simeq \mathcal{L}_{1, a}^{3} \simeq \mathbb{k}_{-a} \otimes \mathcal{L}_{1,1}^{3}$, for all $a \in \mathbb{k}^{\times}$. 
4.3.2. Tensor product of 3-dimensional indecomposable $K$-modules. Let $u_{i j}:=$ $e_{i} \otimes e_{j}, i, j \in \mathbb{I}_{3}$. Consider the basis $\mathfrak{B}=\left\{v_{i}: i \in \mathbb{I}_{9}\right\}$ of $\mathcal{L}_{2,1}^{3} \otimes \mathcal{L}_{2,1}^{3}$, where

$$
\begin{aligned}
& v_{1}=u_{31}, \quad v_{2}=-u_{32}, \quad v_{3}=u_{13}, \quad v_{4}=-u_{23}, \quad v_{5}=u_{33}, \\
& v_{6}=\frac{1}{2}\left(u_{12}-u_{21}\right), \quad v_{7}=2 u_{11}, \quad v_{8}=u_{12}+u_{21}, \quad v_{9}=-u_{12}+u_{22},
\end{aligned}
$$

which give us the normal Jordan form of $g$. In the basis $\mathfrak{B}$, the actions of the $g, x_{1}$ and $x_{2}$ are determined by (4.19) where

$$
\begin{aligned}
& A=\left(\begin{array}{cc}
\mathrm{J}_{2}(-1) & 0 \\
0 & \mathrm{~J}_{2}(-1)
\end{array}\right), \quad B=\left(\begin{array}{cc}
\mathrm{id}_{2} & 0 \\
0 & \mathrm{~J}_{3}(1)
\end{array}\right), \\
& C=\left(\begin{array}{lllll}
-e_{1}+e_{3} & 0 & 0 & 0 & 0
\end{array}\right), \quad E=\left(\begin{array}{llllll}
-e_{2}+e_{4} & 0 & 0 & 0 & 0
\end{array}\right) \text {, } \\
& D=\frac{1}{2}\left(\begin{array}{c}
0 \\
-2\left(e_{2}+e_{4}\right)^{t} \\
\left(e_{1}+e_{3}-e_{4}\right)^{t} \\
-\left(e_{2}-e_{4}\right)^{t} \\
0
\end{array}\right), \quad F=\left(\begin{array}{c}
0 \\
\left(e_{1}+e_{2}-e_{3}+2 e_{4}\right)^{t} \\
0 \\
-\frac{1}{2}\left(e_{1}-e_{2}+e_{3}-2 e_{4}\right)^{t} \\
\left(e_{2}+e_{4}\right)^{t}
\end{array}\right) \text {. }
\end{aligned}
$$

Proposition 4.11. $T=\mathcal{L}_{2,1}^{3} \otimes \mathcal{L}_{2,1}^{3}$ is an indecomposable $K$-module.

Proof. Suppose that $T=U \oplus W$, with $U$ and $W$ non-trivial submodules of $T$. Note that $\operatorname{ker} x_{2}^{2}=\operatorname{ker} x_{21}=\operatorname{ker} x_{2} x_{1}=T-\left\langle v_{5}\right\rangle, x_{21} T=\left\langle v_{7}\right\rangle, x_{2} x_{1} T=\left\langle v_{6}\right\rangle$ and $x_{2}^{2} T=\left\langle v_{6}, v_{8}\right\rangle$. We can assume $x_{21} U=\{0\}$ and $x_{21} W=\left\langle v_{7}\right\rangle$. Let $u \in U$ and $w \in W$ such that $v_{5}=u+w$. From $\left\langle x_{21} w\right\rangle=\left\langle x_{21} v_{5}\right\rangle=\left\langle v_{7}\right\rangle$ follows that $v_{7} \in W$. Similarly, from $x_{2} x_{1} T=\left\langle v_{6}\right\rangle$ follows $v_{6} \in W$ and from $x_{2}^{2} T=\left\langle v_{6}, v_{8}\right\rangle$ follows $v_{8} \in W$. Thus, $W^{\prime}=\left\langle v_{6}, v_{7}, v_{8}\right\rangle \subset W$. Since $x_{1} u \in W^{\prime}$, we obtain $u \in \operatorname{ker} x_{1}=\left\langle v_{1}-v_{3}, v_{6}, v_{7}, v_{8}, v_{9}\right\rangle$. Hence, $x_{2} u \in\left\langle v_{6}\right\rangle$ and whence $u \in \operatorname{ker} x_{2}=\left\langle v_{6}, v_{7}, v_{8}, v_{9}\right\rangle$. Since $u \notin W^{\prime}$, it follows that $(g-\mathrm{id}) u=\alpha v_{7}+\beta v_{8}$ with $\alpha, \beta \in \mathbb{k}$ and $\beta \neq 0$. Then $0 \neq(g-\mathrm{id}) u \in U \cap W$ which is a contradiction.

Corollary 4.12. Let $a, b \in \mathbb{k}^{\times}$. Then

(i) $\mathcal{L}_{2, a}^{3} \otimes \mathcal{L}_{2, b}^{3}$ is an indecomposable $K$-module.

(ii) $\mathcal{L}_{1, a}^{3} \otimes \mathcal{L}_{1, b}^{3}$ is an indecomposable $K$-module.

Proof. By Remark 4.10, $\mathcal{L}_{2, a}^{3} \otimes \mathcal{L}_{2, b}^{3} \simeq \mathbb{k}_{a b} \otimes \mathcal{L}_{2,1}^{3} \otimes \mathcal{L}_{2,1}^{3}$. Hence (i) follows from Proposition 4.11, By Remark 4.10, $\mathcal{L}_{1, a}^{3} \otimes \mathcal{L}_{1, b}^{3} \simeq\left(\mathcal{L}_{2, a^{-1}}^{3}\right)^{*} \otimes\left(\mathcal{L}_{2, b^{-1}}^{3}\right)^{*} \simeq$ $\left(\mathcal{L}_{2, b^{-1}}^{3} \otimes \mathcal{L}_{2, a^{-1}}^{3}\right)^{*}$. Thus, (ii) follows from (i).

Fix a basis $\left\{e_{1}, e_{2}, e_{3}\right\}$ of $\mathcal{L}_{2,1}^{3}$, a basis $\left\{\tilde{e}_{1}, \tilde{e}_{2}, \tilde{e}_{3}\right\}$ of $\mathcal{L}_{1,1}^{3}$ and $u_{i j}:=e_{i} \otimes \tilde{e}_{j}$, $i, j \in \mathbb{I}_{3}$. As above, the basis $\mathfrak{B}=\left\{v_{i}: i \in \mathbb{I}_{9}\right\}$ of $\mathcal{L}_{2,1}^{3} \otimes \mathcal{L}_{1,1}^{3}$ with

$$
\begin{aligned}
& v_{1}=u_{31}, \quad v_{2}=-u_{32}, \quad v_{3}=u_{13}, \quad v_{4}=-u_{23}, \quad v_{5}=u_{33}, \\
& v_{6}=\frac{1}{2}\left(u_{12}-u_{21}\right), \quad v_{7}=2 u_{11}, \quad v_{8}=u_{12}+u_{21}, \quad v_{9}=-u_{12}+u_{22} \text {, }
\end{aligned}
$$


give us the normal Jordan form of $g$ and the actions of the $g, x_{1}$ and $x_{2}$ are determined by (4.19) where

$$
\begin{aligned}
& A=\left(\begin{array}{cc}
\mathrm{J}_{2}(-1) & 0 \\
0 & \mathrm{~J}_{2}(-1)
\end{array}\right), \quad B=\left(\begin{array}{cc}
\mathrm{id}_{2} & 0 \\
0 & \mathrm{~J}_{3}(1)
\end{array}\right), \\
& C=\left(\begin{array}{lllll}
e_{3} & \frac{1}{2} e_{3} & 0 & e_{3} & -e_{4}
\end{array}\right), \quad E=\left(\begin{array}{lllll}
e_{4} & -\frac{1}{2}\left(e_{3}-e_{4}\right) & 2 e_{3} & e_{3}-e_{4} & 0
\end{array}\right), \\
& D=\frac{1}{2}\left(\begin{array}{c}
2 e_{2}^{t} \\
-2 e_{2}^{t} \\
e_{1}^{t} \\
-e_{2}^{t} \\
0
\end{array}\right), \quad F=\left(\begin{array}{c}
-e_{1}^{t} \\
\left(e_{1}+e_{2}\right)^{t} \\
0 \\
-\frac{1}{2}\left(e_{1}-e_{2}\right)^{t} \\
e_{2}^{t}
\end{array}\right) .
\end{aligned}
$$

Proposition 4.13. $\mathcal{L}_{2,1}^{3} \otimes \mathcal{L}_{1,1}^{3} \simeq U \oplus W$, where $U$ is an indecomposable $K$-module of dimension 8 and $W=\mathbb{k}_{1}$.

Proof. Consider $\mathfrak{C}=\left\{v_{1}, v_{2}, v_{3}, v_{4}, v_{5}-v_{6}, v_{7}, v_{8}, v_{6}+v_{9}\right\}$ which is a linearly independent set. Notice that $U=\langle\mathfrak{C}\rangle$ and $W=\left\langle v_{5}-2 v_{6}-\frac{1}{2} v_{7}\right\rangle$ are $K$ submodules of $\mathcal{L}_{2,1}^{3} \otimes \mathcal{L}_{1,1}^{3}$. Moreover $\mathcal{L}_{2,1}^{3} \otimes \mathcal{L}_{1,1}^{3} \simeq U \oplus W$ and $W=\mathbb{k}_{1}$.

Let $U_{1}$ and $U_{2}$ be non-trivial $K$-submodules of $U$ with $U=U_{1} \oplus U_{2}$. Suppose that $v_{3} \in U_{1}$ and let $u \in U_{2}$ a vector with coordinates $\left(\lambda_{i}\right), i \in \mathbb{I}_{8}$, in the basis $\mathfrak{C}$. From $x_{2}^{2} u=-\lambda_{1} v_{3} \in U_{1} \cap U_{2}$ we see that $\lambda_{1}=0$. Similarly, applying $x_{2}^{2} g, x_{1}$ and $x_{2}$ on $u$ we conclude that $\lambda_{i}=0$, for all $i \in \mathbb{I}_{8}$. Then $U_{2}=0$, a contradiction. Hence, $v_{3}=u_{1}+u_{2}$ with $u_{i} \in U_{i}$ and $u_{i} \neq 0$ for $i \in \mathbb{I}_{2}$. Thus $u_{1}, u_{2} \in V_{g}^{-1}=\left\langle v_{1}, v_{3}\right\rangle$. Since $v_{3} \notin U_{1}$, it follows that $x_{2}^{2} \cdot u_{1} \neq$ 0 . Then $x_{2}^{2} \cdot u_{1}=-x_{2}^{2} \cdot u_{2} \in\left\langle v_{3}\right\rangle$ and $v_{3} \in U_{1} \cap U_{2}$, a contradiction.

Remark 4.14. Since $\operatorname{dim} U^{ \pm 1}=2, U \neq \mathcal{L}_{i, a, b}^{8}$, for any $a \in \mathbb{k}^{\times}, b \in \mathbb{k}, i \in \mathbb{I}_{2}$.

\section{REFERENCES}

[AAH1] N. Andruskiewitsch, I. Angiono and I. Heckenberger. Liftings of Jordan and super Jordan planes. Proc. Edinb. Math. Soc., II. Ser., to appear.

[AAH2] N. Andruskiewitsch, I. Angiono and I. Heckenberger. On finite GK-dimensional Nichols algebras over abelian groups. Mem. Amer. Math. Soc., to appear.

$[\mathrm{ABDF}]$ N. Andruskiewitsch, D. Bagio, S. Della Flora and D. Flôres. Representations of the super Jordan plane. São Paulo J. Math. Sci. 11, 312-325 (2017).

[I] N. Iyudu, Representation Spaces of the Jordan Plane. Comm. Algebra. 42 (8), 3507-3540 (2014).

[RS] S. Reca and A. Solotar, Homological invariants relating the super Jordan plane to the Virasoro algebra. J. Algebra. 507, 120-185 (2018).

[S] B. Srinivasan, The modular representation ring of a cyclic p-group. Proceedings of the London Mathematical Society. 14, 677-688 (1964).

Famaf-Universidad Nacional de Córdoba, Ciem (CONiCET), Medina Allende s/n, Ciudad Universitaria, 5000 Córdoba, República Argentina.

E-mail address: andrus@famaf .unc.edu.ar

Departamento de Matemática, Universidade Federal de Santa Maria, 97105900, SANTA Maria, RS, BraziL

E-mail address: bagio@smail.ufsm.br, saradia.flora@ufsm.br, flores@ufsm.br 